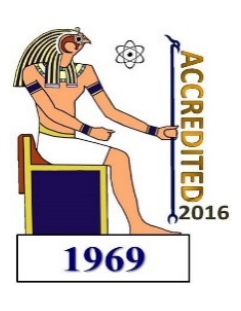

Delta Journal of Science

Available online at

https://djs.journals.ekb.eg/

Research Article

CHEMISTRY

\title{
Some Chemical Aspects of 1,2,4-triazine derivatives
}

\author{
A. A. El-Barbary A. M. Sharaf,Bader E-Bader \\ Chemistry Department, Faculty of Science, Tanta University, Egypt \\ Corresponding author e-mail: aeelbarbary@hotmail.com
}

\begin{abstract}
Condensation of 3a,b with some aromatic aldehydes afforded the Schiff bases (4a-f). Reaction of 3a,b with aromatic aldehydes and alkyl phosphites gave the corresponding amino phosphonates (5a-d). Heating 3a,b with $\mathbf{6 a - f}$ in $\mathrm{POCl}_{3}$ at $85^{\circ} \mathrm{C}$ furnished the amino sulphonamides (7a- $\left.\mathbf{l}\right)$. Acid hydrolysis of compounds $7 \mathbf{a}, \mathbf{b}$ and $\left.7 \mathbf{k}\right)$ furnished the amines $(\mathbf{8 a}-\mathbf{c})$. Condensation of 3a, b with 9a,b afforded the cyclized product (10a,b). Reaction of 3a with halo esters afforded 12 and 13. Heating 3a,b with 14a-c afforded 15a-f. Hydrolysis of 15a,b afforded 16a,b. Fusion of 3a,b with 1,4-butane sultone gave sultams $(\mathbf{1 8 a}, \mathbf{b})$. The antimicrobial activity o9f some products was tried
\end{abstract}

Key words: 1, 2, 4-triazines, aldehydes, aminophosphonates, sultams, biological activity.

\section{Introduction:}

1,2,4-Triazines were known to have many biological activities as antihypertensive, ${ }^{1}$ anticonvulsant agents, ${ }^{2}$ antiinflamatory, ${ }^{3}$ muscle relaxants ${ }^{4}$ and antibacterial agents. $^{5}$. As we occupied several years ago with the chemistry of 1,2,4-triazines, we felt prompt that preparation of new type of these compounds have the triazine moiety gives at a diverse biological activity. So, it is our goal to extend our study in this direction to explore their reactivity towards different reagents to synthesize some new derivatives for testing their biological activates.

\section{RESULTS AND DISCUSSION}

Refluxing benzaldehyde with hippuric acid in acetic anhydride in presence of sodium acetate anhydrous for 2 $\mathrm{hr}$ afforded 4-benzyl-2-phenyloxazol-5(2H)-one (1), ${ }^{6}$ which on boiling in sodium hydroxide solution for $3 \mathrm{hr}$, afforded phenylpyruvic acid (2), ${ }^{7}$ which on boiling in $80 \%$ aqueous methanol with thiocarbohydrazide for $5 \mathrm{hr}$ afforded (3a) (Scheme 1). Its IR spectrum showed the $(\mathrm{C}=\mathrm{S})$ at $1284 \mathrm{~cm}^{-1}$ and $(\mathrm{C}=\mathrm{O})$ at $1607 \mathrm{~cm}^{-1}$, its ${ }^{1} \mathrm{H}-\mathrm{NMR}$ spectrum showed a singlet $\left(\mathrm{CH}_{2}\right)$ at $2.41 \mathrm{ppm}$ and its $\mathrm{MS}$ spectrum showed the (EI) $\mathrm{m} / \mathrm{z}$ at 233.9. Compound $\mathbf{3 b}$ was prepared according to literature method. ${ }^{8,9}$

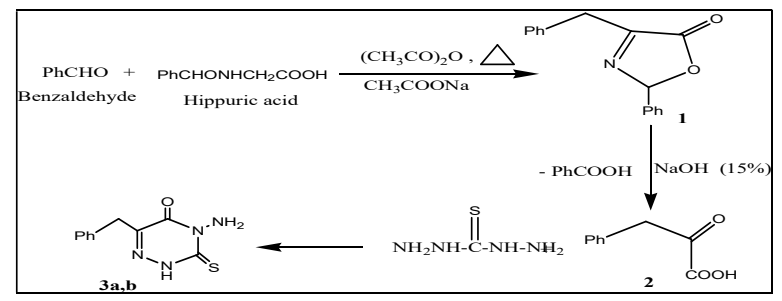

Scheme 1

Condensation of compounds $\mathbf{3 a , b}$ with some aromatic aldehydes namely: benzaldehyde, 4methoxybenzaldehyde and/or 2-nitrobenzaldehyde in boiling glacial acetic acid afforded the corresponding Schiff bases (4a-f) (Scheme 2). The ${ }^{1} \mathrm{H}-\mathrm{NMR}$ spectrum of 4b showed a singlet $\left(3 \mathrm{H}, \mathrm{CH}_{3}\right)$ at 1.80 and a singlet $\mathrm{NH}$ at $8.51 \mathrm{ppm}$. Aminophosphonates are phosphonic analogs of naturally occurring $\alpha$-amino acids and have attracted much attention due to their biological activities and extensive application in organic chemistry. ${ }^{10}$ So, we now report a facile and efficient one step synthesis (one pot) of $\alpha$ - 
aminophosphonate derivatives of 1,2,4-triazines by a three component condensation reaction. Accordingly, compounds $\mathbf{3 a}, \mathbf{b}$ were allowed to react with triethyland/or triphenyl-phosphite and an aromatic aldehyde (either benzaldehyde and/or 4-N-dimethyl

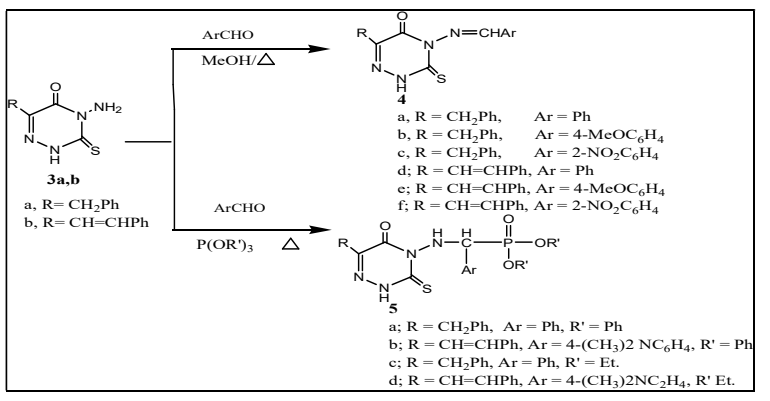

Scheme 2

aminobenzaldehyde) in boiling glacial acetic acid for 5-6 $\mathrm{hr}$ to give the corresponding amino phosphonates (5a-d) in good yields (Scheme 2). The IR spectrum of $\mathbf{5 b}$ showed the $(\mathrm{P}=\mathrm{O})$ at $1250 \mathrm{~cm}^{-1}$, Its ${ }^{1} \mathrm{H}-\mathrm{NMR}$ spectrum showed a singlet $\left(6 \mathrm{H}, 2 \mathrm{CH}_{3}\right)$ at 1.42 and $1.80 \mathrm{ppm}$ and Its $\mathbf{M S}: \mathrm{m} / \mathrm{z}$ at $611.9\left(\mathrm{C}_{32} \mathrm{H}_{30} \mathrm{~N}_{5} \mathrm{O}_{4} \mathrm{PS}, 99 \%, \mathrm{M}^{+}\right)$.

Heating 3a,b with (benzene and/or toluene) sulphonyl amino- (acetic and / or propionic)-acids (6a-f) in phosphorus oxychloride at $85{ }^{\circ} \mathrm{C}$ for $6-8 \mathrm{hr}$ furnished the corresponding heterocyclic amino sulphonamides (7a- I) (Scheme 3). The IR spectrum of compound 7e showed the $\mathrm{C}=\mathrm{O}$ at $1699 \mathrm{~cm}^{-1}$ and $\mathrm{CH}$ at $2914 \mathrm{~cm}^{-1}$, its ${ }^{1} \mathrm{H}-\mathrm{NMR}$ spectrum showed a singlet $\left(3 \mathrm{H}, \mathrm{CH}_{3}\right)$ at 2.41 and a singlet $(1 \mathrm{H}, \mathrm{NH})$ at $7.99 \mathrm{ppm}$. Its MS spectrum showed the (EI) $\mathrm{m} / \mathrm{z}$ at $453.09\left(\mathrm{C}_{21} \mathrm{H}_{19} \mathrm{~N}_{5} \mathrm{O}_{3} \mathrm{~S}_{2}, 4.89 \%, \mathrm{M}^{+}\right)$. The IR spectrum of compound $7 \mathbf{h}$ showed the $\mathrm{CH}_{2}$ at $2861 \mathrm{~cm}^{-1}$ and $\mathrm{CH}$ at $2985 \mathrm{~cm}^{-1}$, its ${ }^{1} \mathrm{H}-\mathrm{NMR}$ spectrum showed a singlet $\left(3 \mathrm{H}, \mathrm{CH}_{3}\right)$ at 1.97 and a singlet $\mathrm{CH}_{2}$ at $4.41 \mathrm{ppm}$ and Its $\mathrm{MS}$ spectrum (EI) showed $\mathrm{m} /$ zat429.09 $\left(\mathrm{C}_{19} \mathrm{H}_{19} \mathrm{~N}_{5} \mathrm{O}_{3} \mathrm{~S}_{2}, 100 \%, \mathrm{M}^{+}\right)$.

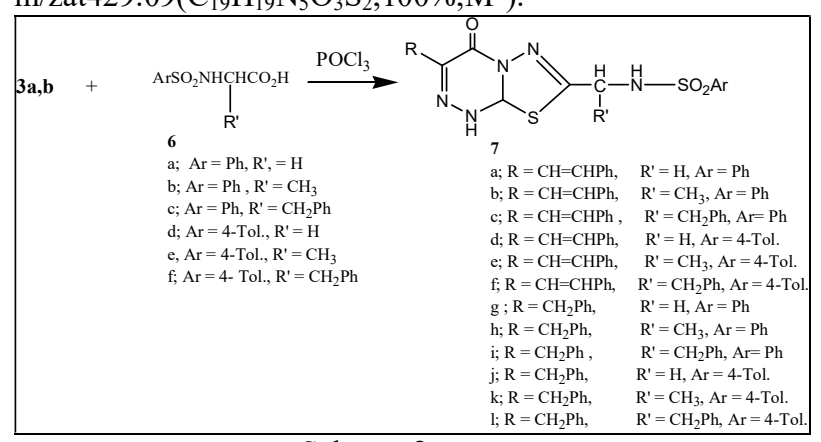

Scheme 3

Acid hydrolysis of compounds (7a,b and $\mathbf{k})$ could be achieved by their refluxing with conc. $\mathrm{HCl}$ for 3-5 hr and led to the formation of new type of amines (8a-c) in addition to 4-toluenesulphonic acid (Scheme 4). The IR spectrum of compound 8a showed the $\mathrm{NH}_{2}$ sym at $3368 \mathrm{~cm}^{-}$ ${ }^{1}$ and $\mathrm{NH}_{2}$ antisym at $3475 \mathrm{~cm}^{-1}$, its ${ }^{1} \mathrm{H}-\mathrm{NMR}$ spectrum showed the single $\mathrm{CH}_{2}$ at 2.35 and a single $\mathrm{NH}_{2}$ at 4.16 ppm. Condensation of compounds 3a, b with 2-(phenyl and/or ethoxy)-1,3-benzoxazin-4H-ones (9a,b) in boiling glacial acetic acid afforded the cyclized product $(\mathbf{1 0 a}, \mathbf{b})$ through the elimination of one molecule of water as the sole product (tlc.) and not

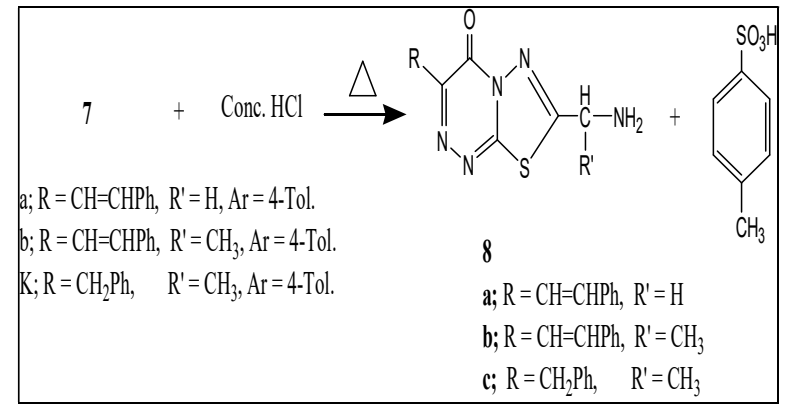

Scheme 4

the open structure N-(3-mercapto-5-oxo-6-styryl-1,2,4triazin-4(5H)-yl)acetamide (11) (Scheme 5). The IR spectrum of compound $\mathbf{1 0 b}$ showed the $\mathrm{NH}$ at $3375 \mathrm{~cm}^{-1}$ and its ${ }^{13} \mathrm{C}$-NMR spectrum showed the $\mathrm{CH}_{3}$ at 14.17, $\mathrm{CH}_{2}$ at 39.29 and $\mathrm{C}=\mathrm{S}$ at $193.50 \mathrm{ppm}$.

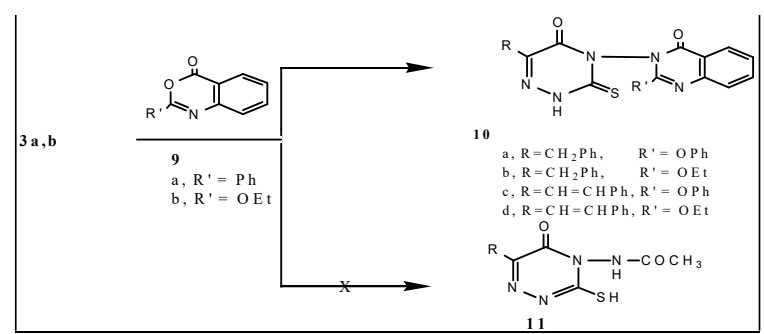

Scheme 5

Reaction of compound 3a with ethyl chloro -(acetate and /or acetoacetate) in boiling methanol in the presence of sodium hydroxide for 3-4 hr afforded the corresponding alkyl derivatives 12 and 13, respectively (Scheme 6). The IR spectrum of compound $\mathbf{1 2}$ showed the $\mathrm{NH}_{2}$ sym at 3452 $\mathrm{cm}^{-1}$ and $\mathrm{NH}_{2 \text { assym. }}$ at $3461 \mathrm{~cm}^{-1}$ and its ${ }^{1} \mathrm{H}-\mathrm{NMR}$ spectrum showed the triplet $\mathrm{CH}_{3}$ at 1.54 and a quartet $\mathrm{CH}_{2}$ at 2.41 ppm. Its IR spectrum of compound 13 showed the $\mathrm{NH}_{2 \mathrm{sym}}$ at $3338 \mathrm{~cm}^{-1}$ and $\mathrm{NH}_{2}$ assym. at $3453 \mathrm{~cm}^{-1}$ and its ${ }^{1} \mathrm{H}-\mathrm{NMR}$ spectrum showed the triplet $\mathrm{CH}_{3}$ at 1.60 , a quartet $\mathrm{CH}_{2}$ at 2.50 and a single $\mathrm{CH}$ at $8.33 \mathrm{ppm}$

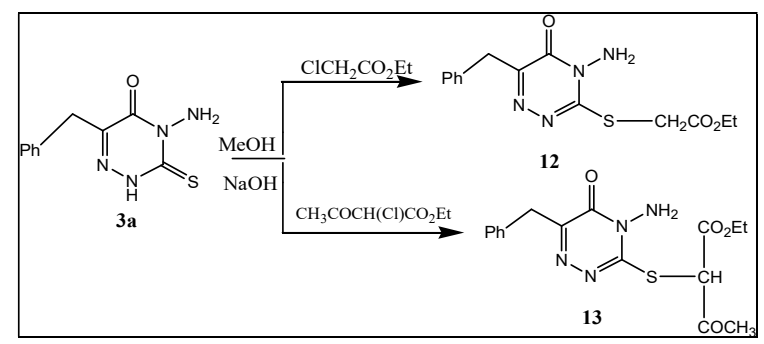

Scheme 6

Heating a mixture of 3a,b with (1,3-dioxo-1,3dihydroisoindol-2-yl)(methyl-, phenyl- and / or benzyl)acetic acid (14a-c) ${ }^{11}$ in phosphorus oxychloride at $85^{\circ} \mathrm{C}$ for 5-6 hr yielded 15a-f after cooling and neutralization 
with $1 \mathrm{~N} \mathrm{NaOH}$ (Scheme 7). The IR spectrum of compound 15b showed the disappearance of $\mathrm{NH}_{2}$ at 3453 $\mathrm{cm}^{-1}$ and the presence of $\mathrm{CH}_{3}$ at $2925 \mathrm{~cm}^{-1}$, its ${ }^{1} \mathrm{H}-\mathrm{NMR}$ spectrum showed the single $\mathrm{CH}_{3}$ at $2.31 \mathrm{ppm}$. The MS spectrum of compound $\mathbf{1 5 f}$ showed the (EI) $\mathrm{m} / \mathrm{z}$ at 493.12 $\left(\mathrm{C}_{27} \mathrm{H}_{19} \mathrm{~N}_{5} \mathrm{O}_{3} \mathrm{~S}, 1.41 \%, \mathrm{M}^{+}\right)$.

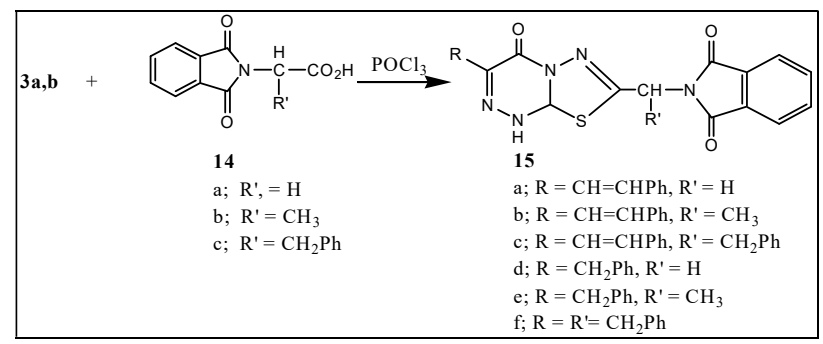

Scheme 7

Acid hydrolysis of $\mathbf{1 5 a , b}$ could be achieved by their refluxing with conc. $\mathrm{HCl}$ for $8-10 \mathrm{hr}$ and led to the formation of a new type of amine 16a,b in addition to phthalic acid (Scheme 8). The IR spectrum of compound 16a showed the presence of $\mathrm{NH}_{2}$ sym at $3368 \mathrm{~cm}^{-1}$ and $\mathrm{NH}_{2}$ antisym at $3475 \mathrm{~cm}^{-1}$. The MS spectrum of compound $\mathbf{1 6 b}$ showed the $(E I) \mathrm{m} / \mathrm{z}$ at $299.08\left(\mathrm{C}_{14} \mathrm{H}_{13} \mathrm{~N}_{5} \mathrm{OS}, 100 \%, \mathrm{M}^{+}\right)$.

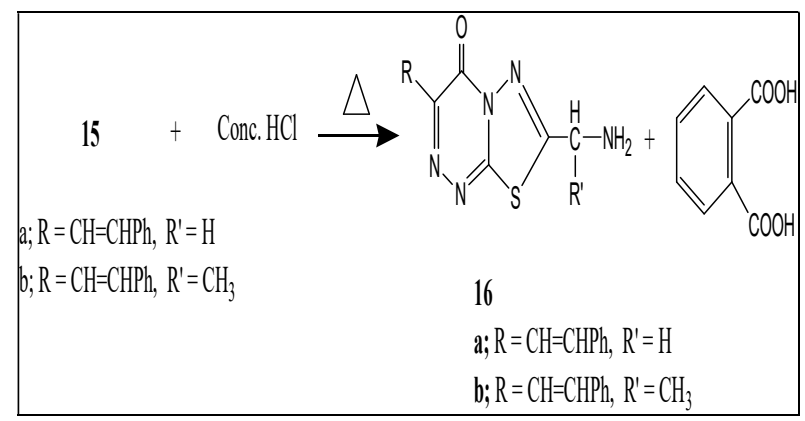

Scheme 8

Fusion of compounds 3a,b with 1,4-butanesultone (17) at $180{ }^{\circ} \mathrm{C}$ for $6-8 \mathrm{hr}$ afforded the corresponding sultams (18a,b), respectively (Scheme 9). The IR spectrum of compound 18a showed the appearance of $\mathrm{S}=\mathrm{O}$ at 1162 $\mathrm{cm}^{-1}$ and $1351 \mathrm{~cm}^{-1}$. The MS spectrum of compound $\mathbf{1 8 b}$ showed the (EI) $\mathrm{m} / \mathrm{z}$ at $364.07\left(\mathrm{C}_{15} \mathrm{H}_{16} \mathrm{~N}_{4} \mathrm{O}_{3} \mathrm{~S}_{2}, 80.12 \%\right.$, $\mathrm{M}^{+}$.

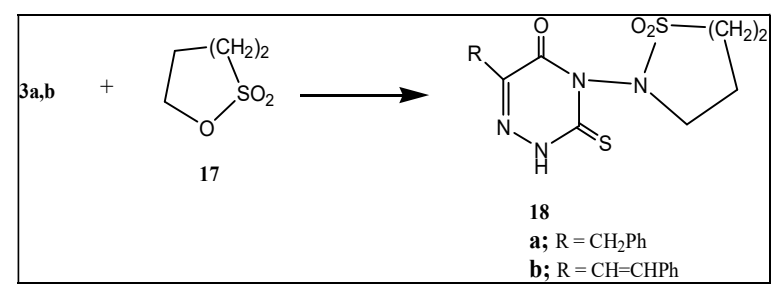

Scheme 9

\section{EXPERIMENTAL}

All melting points were uncorrected and performed by the open capillary melting point apparatus. Microanalyses performed by Microanalysis Unit, Central Laboratory, Tanta University, Tanta, Egypt. IR spectra were recorded with a perkin-Elmer 1720 spectrometer. The NMR spectra were recorded on a Bruker AC 250 FT NMR spectrometer at $250 \mathrm{MHz}$ for ${ }^{1} \mathrm{H}$ and $62.9 \mathrm{MHz}$ for ${ }^{13} \mathrm{C}$,Varian UNITY $500 \mathrm{NMR}$ spectrometer at $500 \mathrm{MHz}$ for ${ }^{1} \mathrm{H}$ or $125.7 \mathrm{MHz}$ for ${ }^{13} \mathrm{C}$, Bruker $200 \mathrm{MHz}$ And Bruker $90 \mathrm{MHz}$ spectrometer using TMS as an internal standard, DMSO and $\mathrm{CHCl}_{3}$ as solvents. Chemical shifts $(\delta)$ are reported in parts per million (ppm) and signals are expressed as s (singlet), $d$ (doublet), $t$ (triplet), q (quartet), m (multiplet) or br (broad). Mass spectra (MS) were recorded using electron ionization (E.I.) on a Varian Mat 311A spectrometer.

Preparation of 4-amino-6-benzyl-3-thioxo-3,4dihydro-1,2,4-triazin-5(2H)-one (3a).

Compound 4-benzyl-2-phenyloxazol-5(2H)-one (1) ${ }^{6}$ and phenylpyruvic acid (2) ${ }^{7}$ were prepared according to known methods. Refluxing 2 (1.3 g, $0.01 \mathrm{~mol})$ in aqueous methanol $80 \%$ with thiocarbohydrazide $(1.06 \mathrm{~g}, 0.01 \mathrm{~mol})$ for $5 \mathrm{hr}$ (tlc.), cooled the reaction mixture at $\mathrm{r}$. $\mathrm{t}$. The solid obtained was filtered off, dried and recrystallized from ethanol to afford (3a) yield: $74 \%$, m.p. $195^{\circ} \mathrm{C}$.

\section{4-Amino-6-benzyl-3-thioxo-3,4-dihydro-1,2,4-triazin- 5(2H)-one (3a).}

IR $(\mathrm{KBr}): v\left(\mathrm{~cm}^{-1}\right)=1284(\mathrm{C}=\mathrm{S}), 1748(\mathrm{C}=\mathrm{O}), 3287\left(\mathrm{NH}_{2}\right.$ asym.), 3446 ( $\mathrm{NH}_{2}$ sym.). ${ }^{1} \mathbf{H}-\mathrm{NMR}$ (DMSO-d $)=\delta 2.41(\mathrm{~s}$, $\left.2 \mathrm{H}, \mathrm{CH}_{2}\right), 3.84\left(\mathrm{~s}, 2 \mathrm{H}, \mathrm{NH}_{2}\right), 9.92(\mathrm{~s}, 1 \mathrm{H}, \mathrm{NH}), 7.88-8.71$ $\left(\mathrm{m}, 5 \mathrm{H}, \mathrm{H}_{\mathrm{ar}}\right)$ ppm. ${ }^{13} \mathbf{C}-\mathbf{N M R}\left(\mathrm{DMSO}-d_{6}\right)=\delta 24.23$ $\left(\mathrm{CH}_{2}\right), 127.38,128.65,129,73,134.28$ and $142.16\left(\mathrm{C}_{\mathrm{ar}}\right)$, $176.87\left(\mathrm{C}=\mathrm{O}_{\text {cyclic }}\right), 181.47\left(\mathrm{C}=\mathrm{S}_{\text {cyclic }}\right)$ ppm. MS (EI): $m / z$ $233.9\left(\mathrm{C}_{10} \mathrm{H}_{10} \mathrm{~N}_{4} \mathrm{OS}, 90.6 \%, \mathrm{M}^{+}\right)$.

Compound $\mathbf{3 b}$ was prepared according to literature method. ${ }^{8,9}$

Condensation of compounds $3 \mathrm{a}, \mathrm{b}$ with some aromatic aldehydes. Formation of Schiff bases (4a-f).

The compounds 3a or $3 \mathbf{b}(0.01 \mathrm{~mol})$ and some aromatic aldehydes namely: benzaldehyde, 4methoxybenzaldehyde and/or 2-nitro- benzaldehyde $(0.01 \mathrm{~mol})$ were mixed. The reaction mixture was refluxed in glacial acetic acid $(15 \mathrm{ml})$ for 4-6 hr (tlc), cooled to $\mathrm{r}$. $\mathrm{t}$. and poured onto ice water. The solid obtained was filtered off, dried and recrystallized from DMF/water to afforded the corresponding Schiff bases (4a-f).

6-Benzyl-4-(benzylideneamino)-3-thioxo-3,4-dihydro1,2,4-triazin-5(2H)-one (4a).

IR (KBr): v $\left(\mathrm{cm}^{-1}\right)=1350(\mathrm{C}=\mathrm{S}), 1605(\mathrm{C}=\mathrm{N}), 1640$ $(\mathrm{C}=\mathrm{O}), 3007(\mathrm{Ph} \mathrm{CH}), 3203(\mathrm{NH}) .{ }^{\mathbf{1}} \mathbf{H}-\mathbf{N M R}\left(\mathrm{CDCl}_{3}\right)=\delta$ $3.81\left(\mathrm{~s}, 2 \mathrm{H}, \mathrm{CH}_{2}\right), 4.63\left(\mathrm{~s}, 1 \mathrm{H}, \mathrm{CH}_{\mathrm{ar}}\right), 6.85-7.3(\mathrm{~m}, 5 \mathrm{H}$, $\left.\mathrm{H}_{\mathrm{ar}}\right)$, 7.47-8.4 (m, 5H, $\left.\mathrm{H}_{\mathrm{ar}}\right), 8.53(\mathrm{~s}, 1 \mathrm{H}, \mathrm{N}=\mathrm{CH}), 10.12(\mathrm{~s}$, $1 \mathrm{H}, \mathrm{NH}) \mathrm{ppm}$.

4-(4-Methoxybenzylideneamino)-6-benzyl-3-thioxo-3,4dihydro-1,2,4-triazin-5(2H)-one (4b).

IR $(\mathrm{KBr}): \mathrm{v}\left(\mathrm{cm}^{-1}\right)=1246(\mathrm{C}=\mathrm{S}), 1557(\mathrm{C}=\mathrm{N}), 1685$ $(\mathrm{C}=\mathrm{O}), 3066(\mathrm{CH}, \mathrm{Ph}), 3139(\mathrm{NH}) .{ }^{\mathbf{1}} \mathbf{H}-\mathbf{N M R}\left(\mathrm{DMSO}-d_{6}\right)$ 
$=\delta 1.80\left(\mathrm{~s}, 3 \mathrm{H}, \mathrm{CH}_{3}\right), 2.18\left(\mathrm{~s}, 2 \mathrm{H}, \mathrm{CH}_{2}\right), 4.15(\mathrm{~s}, 1 \mathrm{H}$, $\mathrm{N}=\mathrm{CH}), 7.56,7.92\left(\mathrm{~m}, 9 \mathrm{H}, \mathrm{H}_{\text {ar. }}\right), 8.51(\mathrm{~s}, 1 \mathrm{H}, \mathrm{NH}) \mathrm{ppm}$.

4-(2-Nitrobenzylideneamino)-6-benzyl-3-thioxo-3,4dihydro-1,2,4-triazin-5(2H)-one $(4 \mathrm{c})$.

IR $(\mathrm{KBr}): v\left(\mathrm{~cm}^{-1}\right)=1587(\mathrm{C}=\mathrm{N}), 1250(\mathrm{C}=\mathrm{S}), 1695$ $(\mathrm{C}=\mathrm{O}), 3083(\mathrm{CH}, \mathrm{Ph}), 3250(\mathrm{NH}), 1520\left(\mathrm{NO}_{2}\right)$.

4-(Benzylideneamino)-6-styryl-3-thioxo-3,4-dihydro1,2,4-triazin-5(2H)-on (4d).

IR $(\mathrm{KBr}) \cup\left(\mathrm{cm}^{-1}\right)=1250(\mathrm{C}=\mathrm{S}), 1561(\mathrm{C}=\mathrm{N}), 1699$ $(\mathrm{C}=\mathrm{O}), 3074(\mathrm{CH}, \mathrm{Ph}), 3148(\mathrm{NH})$.

4-(2-Nitrobenzylideneamino)-6-styryl-3-thioxo-3,4dihydro-1,2,4-triazin-5(2H)-one (4e).

IR $(\mathrm{KBr}) \quad v\left(\mathrm{~cm}^{-1}\right)=1250(\mathrm{C}=\mathrm{S}), 1545(\mathrm{C}=\mathrm{N}), 1684$ $(\mathrm{C}=\mathrm{O}), 3009(\mathrm{CH}, \mathrm{Ph}), 3274(\mathrm{NH}) .{ }^{\mathbf{1}} \mathbf{H}-\mathbf{N M R}\left(\mathrm{CDCl}_{3}\right)=\delta$ $3.25\left(\mathrm{~s}, 3 \mathrm{H}, \mathrm{CH}_{3}\right), 3.72(\mathrm{~d}, 2 \mathrm{H}, J=2.97 \mathrm{~Hz}, \mathrm{CH}=\mathrm{CH})$, 6.84-7.10 (m, 5H, $\left.\mathrm{H}_{\text {ar. }}\right), 7.42-7.85$ (m, 4H, $\left.\mathrm{H}_{\text {ar. }}\right), 7.25$ (s, $1 \mathrm{H}, \mathrm{N}=\mathrm{CH}), 10.25(\mathrm{~s}, 1 \mathrm{H}, \mathrm{NH}) \mathrm{ppm}$.

4-(4-Methoxybenzylideneamino)-6-styryl-3-thioxo-3,4dihydro-1,2,4-triazin-5(2H)-one (4f).

IR $(\mathrm{KBr}) v\left(\mathrm{~cm}^{-1}\right)=1352(\mathrm{C}=\mathrm{S}), 1489\left(\mathrm{NO}_{2}\right), 1502$ $(\mathrm{C}=\mathrm{N}), 1603(\mathrm{C}=\mathrm{O}), 2920(\mathrm{CH}) .{ }^{1} \mathbf{H}-\mathbf{N M R}\left(\mathrm{DMSO}-d_{6}\right)=$ $\delta 4.03(\mathrm{~s}, 1 \mathrm{H}, \mathrm{CH}), 7.83(\mathrm{~d}, 2 \mathrm{H}, J=3.0 \mathrm{~Hz}, \mathrm{CH}=\mathrm{CH})$, 8.22 - $8.94\left(\mathrm{~m}, 7 \mathrm{H}, \mathrm{H}_{\mathrm{ar}}\right), 11.01(\mathrm{~s}, 1 \mathrm{H}, \mathrm{NH}) \mathrm{ppm}$.

Table 1: Physical and analytical data of compounds $\mathbf{4 a - f}$.

\begin{tabular}{|c|c|c|c|c|c|c|}
\hline Cpd & $\begin{array}{l}\text { M.P } \\
\left({ }^{\circ} \mathrm{C}\right)\end{array}$ & $\begin{array}{l}\text { Yiel } \\
\text { d } \\
(\%)\end{array}$ & $\begin{array}{l}\text { M. F. } \\
\text { (M. W.) }\end{array}$ & \multicolumn{3}{|c|}{$\begin{array}{l}\text { M.A. (\%); } \\
\text { Calcd/Found }\end{array}$} \\
\hline $4 a$ & $\begin{array}{l}244 \\
-6\end{array}$ & 73 & $\begin{array}{l}\mathrm{C}_{17} \mathrm{H}_{14} \mathrm{~N}_{4} \mathrm{OS} \\
(322.38)\end{array}$ & $\begin{array}{l}63.33 \\
62.97\end{array}$ & $\begin{array}{l}4.38 \\
3.92\end{array}$ & $\begin{array}{l}17.38 \\
16.94\end{array}$ \\
\hline $4 b$ & $\begin{array}{l}271 \\
-3\end{array}$ & 93 & $\begin{array}{l}\mathrm{C}_{18} \mathrm{H}_{16} \mathrm{~N}_{4} \mathrm{O}_{2} \mathrm{~S} \\
(352.41)\end{array}$ & $\begin{array}{l}61.35 \\
61.06\end{array}$ & $\begin{array}{l}4.58 \\
4.22\end{array}$ & $\begin{array}{l}15.90 \\
15.72\end{array}$ \\
\hline $4 c$ & $\begin{array}{l}264 \\
-6\end{array}$ & 72 & $\begin{array}{l}\mathrm{C}_{17} \mathrm{H}_{13} \mathrm{~N}_{5} \mathrm{O}_{3} \mathrm{~S} \\
(367.38)\end{array}$ & $\begin{array}{l}55.58 \\
55.17\end{array}$ & $\begin{array}{l}3.57 \\
3.31\end{array}$ & $\begin{array}{l}19.06 \\
18.84\end{array}$ \\
\hline 4d & $\begin{array}{l}302 \\
-4\end{array}$ & 53 & $\begin{array}{l}\mathrm{C}_{18} \mathrm{H}_{14} \mathrm{~N}_{4} \mathrm{OS} \\
(334.39)\end{array}$ & $\begin{array}{l}64.65 \\
64.24\end{array}$ & $\begin{array}{l}4.22 \\
3.93\end{array}$ & $\begin{array}{l}16.75 \\
16.53\end{array}$ \\
\hline $4 e$ & $\begin{array}{l}283 \\
-5\end{array}$ & 74 & $\begin{array}{l}\mathrm{C}_{19} \mathrm{H}_{16} \mathrm{~N}_{4} \mathrm{O}_{2} \mathrm{~S} \\
(364.42)\end{array}$ & $\begin{array}{l}62.62 \\
62.37\end{array}$ & $\begin{array}{l}4.43 \\
3.99\end{array}$ & $\begin{array}{l}15.37 \\
15.14\end{array}$ \\
\hline 4f & $\begin{array}{l}295 \\
-7\end{array}$ & 82 & $\begin{array}{l}\mathrm{C}_{18} \mathrm{H}_{13} \mathrm{~N}_{5} \mathrm{O}_{3} \mathrm{~S} \\
(379.39)\end{array}$ & $\begin{array}{l}56.98 \\
56.79\end{array}$ & $\begin{array}{l}3.45 \\
3.14\end{array}$ & $\begin{array}{l}18.46 \\
18.12\end{array}$ \\
\hline
\end{tabular}

Reaction of compounds $3 \mathrm{a}, \mathrm{b}$ with some aromatic aldehydes and triethyl- and / or triphenyl-phosphite. Formation of 5a-d

A mixture of 3a,b $(0.01 \mathrm{~mol})$, some aromatic aldehydes namely: benzaldehyde and/or 4-N-dimethyl aminobenzaldehyde $)^{12}(0.01 \mathrm{~mol})$ and triethyl- and/or triphenyl-phosphite $(0.02 \mathrm{~mol})$ in glacial acetic acid (30 $\mathrm{ml}$ ) was heated at $100^{\circ} \mathrm{C}$ for $5-6 \mathrm{hr}$ (tlc.). The reaction mixture was concentrated to $1 / 4$ volume and poured onto ice. The solid formed was filtered off, washed by petroleum ether followed by recrystallization from methanol to give the corresponding amino phosphonates (5a-d) in good yields (Scheme 2). The data are listed in Table 2.

Diphenyl(6-benzyl-5-oxo-3-thioxo-2,3-dihydro-1,2,4triazin-4(5H)-ylimino)-(phenyl)methylphosphonate (5a).

IR $(\mathrm{KBr}) \cup\left(\mathrm{cm}^{-1}\right)=1224(\mathrm{C}=\mathrm{S}), 1305(\mathrm{P}=\mathrm{O}), 1641$ $(\mathrm{C}=\mathrm{N}), 1708(\mathrm{C}=\mathrm{O}), 2256\left(\mathrm{CH}_{2}\right), 3055\left(\mathrm{CH}_{\mathrm{ar}}\right), 3436$ (NH). ${ }^{1} \mathbf{H}-\mathrm{NMR}\left(\mathrm{DMSO}-d_{6}\right)=\delta 2.51\left(\mathrm{~s}, 1 \mathrm{H}, \mathrm{NH}_{\mathrm{acyclic}}\right)$, 3.45 (s, 1H, CHPh), 6.08 (s, 2H, $\left.\mathrm{CH}_{2} \mathrm{Ph}\right), 7.1-7.3(\mathrm{~m}, 20 \mathrm{H}$, $\left.\mathrm{H}_{\mathrm{ar}}\right), 7.62\left(\mathrm{~s}, 1 \mathrm{H}, \mathrm{NH}_{\text {cyclic }}\right) \mathrm{ppm}$.

Diphenyl(4-(dimethylamino)phenyl)(5-oxo-6-styryl-3thioxo-2,3-dihydro-1,2,4-triazin-4(5H)ylimino)methyIphosphonate (5b).

IR $(\mathrm{KBr}) \cup\left(\mathrm{cm}^{-1}\right)=1197(\mathrm{C}=\mathrm{S}), 1308(\mathrm{P}=\mathrm{O}), 1631$ $(\mathrm{C}=\mathrm{N}), 1723(\mathrm{C}=\mathrm{O}), 2981(\mathrm{CH}), 3086\left(\mathrm{CH}_{\mathrm{ar}}\right), 3427(\mathrm{NH})$. ${ }^{1}$ H-NMR $\left(\mathrm{DMSO}-d_{6}\right)=\delta 1.35\left(\mathrm{~s}, 6 \mathrm{H}, 2 \mathrm{CH}_{3}\right), 2.60(\mathrm{~s}, 1 \mathrm{H}$, $\left.\mathrm{NH}_{\text {acyclic }}\right), 3.65$ (s, 1H, CHPh), 6.55-7.05 (d, 2H, $J=2.85$ $\mathrm{Hz}, \mathrm{CH}=\mathrm{CH}), 7.5-8.8\left(\mathrm{~m}, 19 \mathrm{H}, \mathrm{H}_{\mathrm{ar}}\right), 9.15\left(\mathrm{~s}, 1 \mathrm{H}, \mathrm{NH}_{\text {cyclic }}\right)$ ppm. MS (EI) $\mathrm{m} / \mathrm{z}=611.9\left(\mathrm{M}^{+}, \mathrm{C}_{32} \mathrm{H}_{30} \mathrm{~N}_{5} \mathrm{O}_{4} \mathrm{PS}, 99 \%\right)$.

Diethyl(6-benzyl-5-oxo-3-thioxo-2,3-dihydro-1,2,4triazin-4(5H)-ylamino)-

(phenyl)methylphosphonate (5c).

IR $(\mathrm{KBr}) \cup\left(\mathrm{cm}^{-1}\right)=1250(\mathrm{C}=\mathrm{S}), 1350(\mathrm{P}=\mathrm{O}), 1520$ $(\mathrm{C}=\mathrm{N}), 1640(\mathrm{C}=\mathrm{O}), 2951\left(\mathrm{CH}_{2}\right), 3057\left(\mathrm{CH}_{\mathrm{ar}}\right), 3465$ $(\mathrm{NH}) .{ }^{1} \mathbf{H}-\mathrm{NMR}\left(\mathrm{DMSO}-d_{6}\right)=\delta 2.51(\mathrm{t}, 6 \mathrm{H}, J=4.12 \mathrm{~Hz}$, $\left.2 \mathrm{CH}_{3}\right) 2.72\left(\mathrm{~s}, 1 \mathrm{H}, \mathrm{NH}_{\text {acyclic }}\right), 3.72(\mathrm{~s}, 1 \mathrm{H}, \mathrm{CHPh}), 4.52$ (q, $\left.4 \mathrm{H}, J=2.43 \mathrm{~Hz}, 2 \mathrm{CH}_{2}\right), 5.26\left(\mathrm{~s}, 2 \mathrm{H}, \mathrm{CH}_{2} \mathrm{Ph}\right), 7.33-9.27$ (m, 10H, $\left.\mathrm{H}_{\mathrm{ar}}\right), 9.72\left(\mathrm{~s}, 1 \mathrm{H}, \mathrm{NH}_{\text {cyclic }}\right) \mathrm{ppm}$.

Diethyl(4-(dimethylamino)phenyl)(5-oxo-6-styryl-3thioxo-2,3-di-hydro-1,2,4-triazin-4(5H)ylamino)methylphosphonate (5d).

IR $(\mathrm{KBr}) v\left(\mathrm{~cm}^{-1}\right)=1222(\mathrm{C}=\mathrm{S}), 1337(\mathrm{P}=\mathrm{O}), 1520$ $(\mathrm{C}=\mathrm{N}), 1641(\mathrm{C}=\mathrm{O}), 2961(\mathrm{CH}), 3087\left(\mathrm{CH}_{\mathrm{ar}}\right), 3152(\mathrm{NH})$. ${ }^{1} \mathbf{H}-\mathbf{N M R}\left(\mathrm{DMSO}-d_{6}\right)=\delta 1.81\left(\mathrm{t}, 6 \mathrm{H}, J=4.13 \mathrm{~Hz}, 2 \mathrm{CH}_{3}\right)$, $2.23\left(\mathrm{~s}, 1 \mathrm{H}, \mathrm{NH}_{\text {acyclic }}\right), 2.53\left(\mathrm{~s}, 6 \mathrm{H}, 2 \mathrm{CH}_{3}, \mathrm{~N}\left(\mathrm{CH}_{3}\right)_{2}\right), 2.55$ $(\mathrm{s}, 1 \mathrm{H}, \mathrm{CHPh}), 4.22\left(\mathrm{q}, 4 \mathrm{H}, J=2.81 \mathrm{~Hz}, 2 \mathrm{CH}_{2} \mathrm{CH}_{3}\right), 7.55-$ $7.61(\mathrm{~d}, 2 \mathrm{H}, J=3.10 \mathrm{~Hz}, \mathrm{CH}=\mathrm{CH}), 7.7-7.9\left(\mathrm{~m}, 9 \mathrm{H}, \mathrm{H}_{\mathrm{ar}}\right)$, $8.54\left(\mathrm{~s}, 1 \mathrm{H}, \mathrm{NH}_{\text {cyclic }}\right) .{ }^{13} \mathbf{C}-\mathbf{N M R}\left(\mathrm{DMSO}-d_{6}\right)=\delta 12.81$ $\left(2 \mathrm{CH}_{3}\right), 21.00\left(2 \mathrm{CH}_{3}\right), 39.70(\mathrm{CH}), 126.25,126.65$, 126.84, 129.76, 137.14 and $137.25\left(\mathrm{C}_{\mathrm{ar} .}\right), 165.28$ $\left(\mathrm{C}=\mathrm{O}_{\text {cyclic }}\right), 181.48\left(\mathrm{C}=\mathrm{S}_{\text {cyclic }}\right) \mathrm{ppm}$.

Table 2: Physical and analytical data of compounds 5a-d

\begin{tabular}{|l|l|l|l|l|l|l|}
\hline Cpd & $\begin{array}{l}\text { M.P. } \\
\left({ }^{\circ} \mathrm{C}\right)\end{array}$ & $\begin{array}{l}\text { Yiel } \\
\text { d } \\
(\%)\end{array}$ & $\begin{array}{l}\text { M. F. } \\
(\text { M. W. })\end{array}$ & \multicolumn{3}{|l|}{$\begin{array}{l}\text { M.A. ( \%); } \\
\text { Calcd/Found } \\
\text { C\% }\end{array}$} \\
\hline 5a & $\begin{array}{l}244- \\
6\end{array}$ & 73 & $\begin{array}{l}\mathrm{C}_{29} \mathrm{H}_{25} \mathrm{~N}_{4} \mathrm{O}_{4} \mathrm{PS} \\
(556.57)\end{array}$ & 62.58 & 4.53 & 10.07 \\
& & & 62.61 & 4.64 & 10.21 \\
\hline
\end{tabular}




\begin{tabular}{|l|l|l|l|l|l|l|}
\hline $\mathbf{5 b}$ & $\begin{array}{l}271- \\
3\end{array}$ & 93 & $\begin{array}{l}\mathrm{C}_{32} \mathrm{H}_{30} \mathrm{~N}_{5} \mathrm{O}_{4} \mathrm{PS} \\
(611.65)\end{array}$ & $\begin{array}{l}62.84 \\
62.56\end{array}$ & $\begin{array}{l}4.94 \\
4.72\end{array}$ & $\begin{array}{l}11.45 \\
11.17\end{array}$ \\
\hline $\mathbf{5 c}$ & $\begin{array}{l}264- \\
6\end{array}$ & 72 & $\begin{array}{l}\mathrm{C}_{21} \mathrm{H}_{25} \mathrm{~N}_{4} \mathrm{O}_{4} \mathrm{PS} \\
(460.49)\end{array}$ & 54.77 & 5.47 & 12.17 \\
$5 \mathbf{5 d}$ & $302-$ & 53 & $\begin{array}{l}\mathrm{C}_{24} \mathrm{H}_{30} \mathrm{~N}_{5} \mathrm{O}_{4} \mathrm{PS} \\
(515.56)\end{array}$ & 55.91 & 5.29 & 11.90 \\
\hline
\end{tabular}

Reaction of 3a,b with (benzene- and/or 4-toluene)sulphonyl amino acids. Formation of the amino sulphonamides $(7 \mathbf{a}-\mathbf{I})$.

To a solution of 3a,b $(0.01 \mathrm{~mol})$ in phosphorus oxychloride $(15 \mathrm{ml})$, (benzene- and / or toluene )sulphonyl amino -(acetic and / or propionic)-acids (6a-f) $(0.005 \mathrm{~mol})^{6}$ were added, in portions. The reaction mixture was heated at $85{ }^{\circ} \mathrm{C}$ for $6-8 \mathrm{hr}(\mathrm{tlc})$ and cooled. Sodium hydroxide solution $(1 \%, 50 \mathrm{ml})$ was added until a solid product was formed. It was filtered off, washed with water, dried and recrystallized from methanol and furnished the corresponding amino sulphonamides (7a-l).

N-((4-Oxo-3-styryl-4,8a-dihydro-1H[1,3,4] thiadiazolo[2,3c][1,2,4]-triazin-7-yl)methyl)benzenesulfonamide (7-a).

IR $(\mathrm{KBr}) v\left(\mathrm{~cm}^{-1}\right)=1335,1375\left(\mathrm{SO}_{2}\right), 1592(\mathrm{C}=\mathrm{N}), 1710$ $(\mathrm{C}=\mathrm{O}), 2981(\mathrm{CH}), 3020\left(\mathrm{CH}_{\mathrm{ar} .}\right), 3335(\mathrm{NH}),{ }^{1} \mathbf{H}-\mathbf{N M R}$ $\left(\mathrm{DMSO}-d_{6}\right)=\delta 3.31\left(\mathrm{~s}, 2 \mathrm{H}, \mathrm{CH}_{2}\right), 7.21-7.40(\mathrm{~d}, 2 \mathrm{H}, J=$ $3.02 \mathrm{~Hz}, \mathrm{CH}=\mathrm{CH}), 7.51-8.82\left(\mathrm{~m}, 10 \mathrm{H}, \mathrm{H}_{\mathrm{ar}}\right), 11.73(\mathrm{~s}, 1 \mathrm{H}$, $\mathrm{NH})$ ppm. ${ }^{13} \mathbf{C}-\mathbf{N M R}\left(\mathrm{DMSO}-d_{6}\right)=\delta 34.00\left(\mathrm{CH}_{2}\right)$, 113.94, 116.20, 117.69, 119.81, 128.61, 128.87, 129.40, 130.96, 137.78, 138.54 and $139.02\left(\mathrm{C}_{\mathrm{ar}}\right), 159.80(\mathrm{C}=\mathrm{N})$, $167.08\left(\mathrm{C}=\mathrm{O}_{\text {cyclic. }}\right)$ ppm.

N-(1-(4-Oxo-3-styryl-4,8adihydro1 $H[1,3,4]$ thiadiazolo$[2,3 \mathrm{c}][1,2,4]$ triazin-7-yl)ethyl)benzenesulfonamide (7b).

IR $(\mathrm{KBr}) v\left(\mathrm{~cm}^{-1}\right)=1332,1373\left(\mathrm{SO}_{2}\right), 1553(\mathrm{C}=\mathrm{N}), 1695$ $(\mathrm{C}=\mathrm{O}), 2932(\mathrm{CH}), 3070\left(\mathrm{CH}_{\mathrm{ar}}\right), 3218(\mathrm{NH}) .{ }^{1} \mathbf{H}-\mathbf{N M R}$ $\left(\mathrm{DMSO}-d_{6}\right)=\delta 2.21\left(\mathrm{~s}, 3 \mathrm{H}, \mathrm{CH}_{3}\right), 3.25(\mathrm{~s}, 1 \mathrm{H}, \mathrm{CH}), 7.55-$ $7.63(\mathrm{~d}, 2 \mathrm{H}, J=2.92 \mathrm{~Hz}, \mathrm{CH}=\mathrm{CH}), 7.75-7.82(\mathrm{~m}, 10 \mathrm{H}$, $\left.\mathrm{H}_{\mathrm{ar}}\right) .{ }^{13} \mathrm{C}-\mathrm{NMR}$ (DMSO- $\left.d_{6}\right)=\delta 17.11\left(\mathrm{CH}_{3}\right), 31.15$ $\left(\underline{\mathrm{CH}}_{3} \mathrm{CH}\right), 40.94\left(\underline{\mathrm{CHCH}}_{3}\right), 153.49(\mathrm{C}-7), 159.89$ (C-3), 161.73 (C-4), 126.44, 129.24, 132.52, 139.95, 148.52 $\left(\mathrm{C}_{\mathrm{ar}}\right), 159.02(\mathrm{C}=\mathrm{N}), 169.77\left(\mathrm{C}=\mathrm{O}_{\text {cyclic }}\right) \mathrm{ppm}$.

N-(1-(4-Oxo-3-styryl-4,8a-dihydro-1 $H$ - $[1,3,4]$ thiadiazolo[2,3-c] $[1,2,4]$ triazin-7-yl)-2phenylethyl)benzenesulfonamide $(7 \mathrm{c})$.

IR $(\mathrm{KBr}) v\left(\mathrm{~cm}^{-1}\right)=1345,1368\left(\mathrm{SO}_{2}\right), 1605(\mathrm{C}=\mathrm{N}), 1717$ $(\mathrm{C}=\mathrm{O}), 2978\left(\mathrm{CH}_{2}\right), 3102(\mathrm{CH}), 3189\left(\mathrm{CH}_{\mathrm{ar}}\right), 3319(\mathrm{NH})$ ppm. ${ }^{1} \mathbf{H}-\mathbf{N M R}\left(\mathrm{DMSO}-d_{6}\right)=\delta 2.51\left(\mathrm{~s}, 2 \mathrm{H}, \mathrm{CH}_{2}\right), 4.11(\mathrm{~s}$, $1 \mathrm{H}, \mathrm{CH}), 7.41-7.55(\mathrm{~d}, 2 \mathrm{H}, J=2.43 \mathrm{~Hz}, \mathrm{CH}=\mathrm{CH}), 7.61-$ $7.92\left(\mathrm{~m}, 15 \mathrm{H}, \mathrm{H}_{\mathrm{ar}}\right), 8.55(\mathrm{~s}, 1 \mathrm{H}, \mathrm{NH}) .{ }^{13} \mathbf{C}-\mathbf{N M R}$ (DMSO$\left.d_{6}\right)=\delta 35.62\left(\mathrm{CH}_{2}\right), 64.42(\mathrm{CH}), 124.73,127.25,129.98$, $130.15,131.32,132.84,137.10$ and $139.73\left(\mathrm{C}_{\text {ar. }}\right), 158.15$ $(\mathrm{C}=\mathrm{N}), 167.81\left(\mathrm{C}=\mathrm{O}_{\text {cyclic }}\right) \mathrm{ppm}$.

4-Methyl-N-((4-oxo-3-styryl-4,8a-dihydro-1 $H[1,3,4]$ thiadiazolo[2,3-c] $[1,2,4]$-triazin-7-yl)methyl)benzenesulfonamide (7d).
IR $(\mathrm{KBr}) v\left(\mathrm{~cm}^{-1}\right)=1351,1374\left(\mathrm{SO}_{2}\right), 1613(\mathrm{C}=\mathrm{N}), 1718$ $(\mathrm{C}=\mathrm{O}), 2935(\mathrm{CH}), 3110\left(\mathrm{CH}_{\mathrm{ar} .}\right), 3465(\mathrm{NH}),{ }^{1} \mathbf{H}-\mathbf{N M R}$ $\left(\mathrm{DMSO}-d_{6}\right)=\delta 2.43\left(\mathrm{~s}, 3 \mathrm{H}, \mathrm{CH}_{3}\right), 3.41\left(\mathrm{~s}, 2 \mathrm{H}, \mathrm{CH}_{2}\right)$, 4.50-7.55 (d, $2 \mathrm{H}, J=3.53 \mathrm{~Hz}, \mathrm{CH}=\mathrm{CH}), 7.56-7.90(\mathrm{~m}$, 9H, $\left.\mathrm{H}_{\mathrm{ar}}\right), 8.95(\mathrm{~s}, 1 \mathrm{H}, \mathrm{NH}) .{ }^{13} \mathbf{C}-\mathbf{N M R}\left(\mathrm{DMSO}-d_{6}\right)=\delta$ $19.31\left(\mathrm{CH}_{3}\right), 38.32\left(\mathrm{CH}_{2}\right), 42.69(\mathrm{CH}), 153.19,158.62$, 164.91, 128.16, 131.12, 138.08, 142.78, $147.93\left(\mathrm{C}_{\mathrm{ar}}\right)$, $158.11(\mathrm{C}=\mathrm{N}), 168.93\left(\mathrm{C}=\mathrm{O}_{\text {cyclic }}\right) \mathrm{ppm}$. MS (EI) $\mathrm{m} / \mathrm{z}=$ $439.07\left(\mathrm{M}^{+}, \mathrm{C}_{20} \mathrm{H}_{17} \mathrm{~N}_{5} \mathrm{O}_{3} \mathrm{~S}_{2}, 7.52 \%\right)$.

\section{4-Methyl-N-(1-(4-oxo-3-styryl-4,8a-dihydro-1 $H$ - $[1,3,4]$ thiadiazolo[2,3-c][1,2,4]-triazin-7-yl)ethyl- )benzenesulfonamide (7e).}

IR $(\mathrm{KBr}) v\left(\mathrm{~cm}^{-1}\right)=1333,1372\left(\mathrm{SO}_{2}\right), 1598(\mathrm{C}=\mathrm{N}), 1699$ $(\mathrm{C}=\mathrm{O}), 2914(\mathrm{CH}), 3139\left(\mathrm{CH}_{\mathrm{ar}}\right), 3558(\mathrm{NH}) .{ }^{\mathbf{1}} \mathbf{H}-\mathbf{N M R}$ $\left(\mathrm{DMSO}-d_{6}\right)=\delta 2.45\left(\mathrm{~s}, 3 \mathrm{H}, \mathrm{CH}_{3} \mathrm{CH}\right), 3.21\left(\mathrm{~s}, 3 \mathrm{H}, \mathrm{CH}_{3} \mathrm{Ph}\right)$ $3.42\left(\mathrm{~s}, 1 \mathrm{H}, \mathrm{CHCH}_{3}\right), 7.35-7.45(\mathrm{~d}, 2 \mathrm{H}, \mathrm{J}=2.64 \mathrm{~Hz}$, $\mathrm{CH}=\mathrm{CH})$ 7.72-7.90 (m, 9H, $\left.\mathrm{H}_{\mathrm{ar}}\right), 9.95(\mathrm{~s}, 1 \mathrm{H}, \mathrm{NH}) .{ }^{13} \mathrm{C}-$ NMR $\left(\right.$ DMSO- $\left.d_{6}\right)=\delta 17.10\left(\mathrm{CH}_{3}\right), 31.12\left(\mathrm{CH}_{3} \mathrm{CH}\right)$, $40.93\left(\mathrm{CHCH}_{3}\right), 153.44,159.89,161.78,126.50,129.60$, 137.13, 142.83, $148.28\left(\mathrm{C}_{\mathrm{ar} .}\right), 156.39(\mathrm{C}=\mathrm{N}), 170.12$ $\left(\mathrm{C}=\mathrm{O}_{\text {cyclic }}\right) \quad$ ppm. MS (EI) $\mathrm{m} / \mathrm{z}=453.09 \quad\left(\mathrm{M}^{+}\right.$, $\mathrm{C}_{21} \mathrm{H}_{19} \mathrm{~N}_{5} \mathrm{O}_{3} \mathrm{~S}_{2}, 4.89 \%$ ).

4-Methyl-N-((4-oxo-3-styryl-4,8a-dihydro-1H-[1,3,4]thiadiazolo[2,3-c][1,2,4]-triazin-7-yl)(ph-enyl)methyl)benzenesulfonamide $(7 f)$.

IR $(\mathrm{KBr}) v\left(\mathrm{~cm}^{-1}\right)=1330,1367\left(\mathrm{SO}_{2}\right), 1611(\mathrm{C}=\mathrm{N}), 1705$ $(\mathrm{C}=\mathrm{O}), 2981\left(\mathrm{CH}_{2}\right), 2991(\mathrm{CH}), 3064\left(\mathrm{CH}_{\mathrm{ar}}\right), 3411(\mathrm{NH})$. ${ }^{1} \mathbf{H}-\mathrm{NMR}\left(\mathrm{DMSO}-d_{6}\right)=\delta 1.34\left(\mathrm{~s}, 3 \mathrm{H}, \mathrm{CH}_{3}\right), 2.51(\mathrm{~s}, 2 \mathrm{H}$, $\left.\mathrm{CH}_{2}\right), 4.12(\mathrm{~s}, 1 \mathrm{H}, \mathrm{CH}), 7.40-7.55(\mathrm{~d}, 2 \mathrm{H}, J=3.13 \mathrm{~Hz}$, $\mathrm{CH}=\mathrm{CH}), 7.60-7.92\left(\mathrm{~m}, 14 \mathrm{H}, \mathrm{H}_{\mathrm{ar}}\right), 8.55(\mathrm{~s}, 1 \mathrm{H}, \mathrm{NH}) \mathrm{ppm}$. ${ }^{13}$ C-NMR (DMSO- $\left.d_{6}\right)=\delta 16.20\left(\mathrm{CH}_{3}\right), 38.31\left(\mathrm{CH}_{2}\right)$, $63.25(\mathrm{CH}), 124.50,128.14,129.08,129.85,131.19$, 131.59, 136.70 and $139.49\left(\mathrm{C}_{\mathrm{ar}}\right), 157.75(\mathrm{C}=\mathrm{N}), 169.14$ $\left(\mathrm{C}=\mathrm{O}_{\text {cyclic }}\right) \mathrm{ppm}$.

N-((3-Benzyl-4-oxo-4,8a-dihydro-1H-[1,3,4]-thiadiazolo[2,3-c] $[1,2,4]$-triazin-7-yl)methyl)-benzenesulfonamide (7g).

IR $(\mathrm{KBr}) v\left(\mathrm{~cm}^{-1}\right)=1334,1365\left(\mathrm{SO}_{2}\right), 1617(\mathrm{C}=\mathrm{N}), 1709$ $(\mathrm{C}=\mathrm{O}), 2922\left(\mathrm{CH}_{2}\right), 2922(\mathrm{CH}), 3056\left(\mathrm{CH}_{\mathrm{ar}}\right), 3554(\mathrm{NH})$. ${ }^{1}$ H-NMR $\left(\mathrm{DMSO}-d_{6}\right)=\delta 2.41\left(\mathrm{~s}, 2 \mathrm{H}, \mathrm{CH}_{2} \mathrm{NH}\right), 4.46(\mathrm{~s}$, $\left.2 \mathrm{H}, \mathrm{CH}_{2} \mathrm{Ph}\right), 7.6-7.91\left(\mathrm{~m}, 10 \mathrm{H}, \mathrm{H}_{\mathrm{ar}}\right), 8.95(\mathrm{~s}, 1 \mathrm{H}, \mathrm{NH})$ ppm. ${ }^{13}$ C-NMR (DMSO- $\left.d_{6}\right)=\delta 18.44\left(\mathrm{CH}_{3}\right), 29.52$ $\left(\mathrm{CH}_{2}\right), 42.42(\mathrm{CH}), 154.16,159.72,163.95,126.96$, $129.71,133.33,139.95,148.67\left(\mathrm{C}_{\mathrm{ar}}\right), 158.35(\mathrm{C}=\mathrm{N})$, $169.22\left(\mathrm{C}=\mathrm{O}_{\text {cyclic }}\right) \mathrm{ppm}$.

N-(1-(3-Benzyl-4-oxo-4,8a-dihydro-1H-[1,3,4]-thiadiazolo[2,3-c] $[1,2,4]$ triazin-7-yl)ethyl)benze-neesulfonamide (7h).

IR $(\mathrm{KBr}) v\left(\mathrm{~cm}^{-1}\right)=1337,1372\left(\mathrm{SO}_{2}\right), 1562(\mathrm{C}=\mathrm{N}), 1703$ $(\mathrm{C}=\mathrm{O}), 2861\left(\mathrm{CH}_{2}\right), 2985(\mathrm{CH}), 3092\left(\mathrm{CH}_{\mathrm{ar}}\right), 3235(\mathrm{NH})$. ${ }^{1} \mathbf{H}-N M R\left(D M S O-d_{6}\right)=\delta 2.45\left(\mathrm{~s}, 3 \mathrm{H}, \mathrm{CH}_{3}\right), 2.51(\mathrm{~s}, 1 \mathrm{H}$, $\mathrm{CH}), 3.25\left(\mathrm{~s}, 2 \mathrm{H}, \mathrm{CH}_{2}\right)$ 7.61-7.88 (m, 10H, $\left.\mathrm{H}_{\mathrm{ar}}\right), 8.00$ (s, $1 \mathrm{H}, \mathrm{NH}) \mathrm{ppm} .{ }^{13} \mathbf{C}-\mathbf{N M R}\left(\mathrm{DMSO}-d_{6}\right)=\delta 18.44\left(\mathrm{CH}_{3}\right)$, $29.54\left(\mathrm{CH}_{2}\right), 42.91(\mathrm{CH}), 155.19,158.74,164.15,127.01$, 
129.83, 134.38, 139.40, $149.57\left(\mathrm{C}_{\mathrm{ar} .}\right), 159.23(\mathrm{C}=\mathrm{N})$, $168.93\left(\mathrm{C}=\mathrm{O}_{\text {cyclic }}\right)$ ppm. MS (EI) $\mathrm{m} / \mathrm{z}=429.09\left(\mathrm{M}^{+}\right.$, $\mathrm{C}_{19} \mathrm{H}_{19} \mathrm{~N}_{5} \mathrm{O}_{3} \mathrm{~S}_{2}, 100.0 \%$ ).

N-((3-Benzyl-4-oxo-4,8a-dihydro-1H- $[1,3,4]$ thiadiazol$0[2,3 c][1,2,4]$ triazin-7-yl)(phenyl)methyl)benzenesulfonamide (7i).

IR $(\mathrm{KBr}) v\left(\mathrm{~cm}^{-1}\right)=1338,1360\left(\mathrm{SO}_{2}\right), 1650(\mathrm{C}=\mathrm{N}), 1711$ $(\mathrm{C}=\mathrm{O}), 2843\left(\mathrm{CH}_{2}\right), 3053\left(\mathrm{CH}_{\mathrm{ar}}\right), 3323(\mathrm{NH}) .{ }^{1} \mathbf{H}-\mathbf{N M R}$ $\left(\mathrm{DMSO}-d_{6}\right)=\delta 2.51(\mathrm{~s}, 1 \mathrm{H}, \mathrm{CH}), 3.92\left(\mathrm{~s}, 2 \mathrm{H}, \mathrm{CHCH}_{2}\right)$, $4.20\left(\mathrm{~s}, 2 \mathrm{H}, \mathrm{CH}_{2} \mathrm{Ph}\right), 7.41-7.92\left(\mathrm{~m}, 10 \mathrm{H}, \mathrm{H}_{\mathrm{ar}}\right), 8.55(\mathrm{~s}, 1 \mathrm{H}$, NH) ppm. ${ }^{13} \mathbf{C}-\mathbf{N M R}\left(\mathrm{DMSO}-d_{6}\right)=\delta 39.25\left(\mathrm{CH}_{2}\right), 62.11$ $(\mathrm{CH}), 127.14,128.01,129.23,129.89,130.19,131.56$, 136.57 and $139.84\left(\mathrm{C}_{\text {ar. }}\right), 158.10(\mathrm{C}=\mathrm{N}), 169.03\left(\mathrm{C}=\mathrm{O}_{\text {cyclic }}\right)$ ppm.

N-((3-Benzyl-4-oxo-4,8a-dihydro-1H-[1,3,4]thiadiazolo[2,3c] $[1,2,4]$-triazin-7-yl)methyl)-4-methylbenzenesulfonamide $(7 \mathbf{j})$.

IR $(\mathrm{KBr}) v\left(\mathrm{~cm}^{-1}\right)=1338,1370\left(\mathrm{SO}_{2}\right), 1632(\mathrm{C}=\mathrm{N}), 1705$ $(\mathrm{C}=\mathrm{O}), 2932\left(\mathrm{CH}_{2}\right), 3126\left(\mathrm{CH}_{\text {ar. }}\right), 3398(\mathrm{NH}) .{ }^{1} \mathbf{H}-\mathbf{N M R}$ $\left(\mathrm{DMSO}-d_{6}\right)=\delta 2.51\left(\mathrm{~s}, 3 \mathrm{H}, \mathrm{CH}_{3} \mathrm{Ph}\right), 3.2(\mathrm{~s}, 1 \mathrm{H}, \mathrm{CH})$, 3.35 (s, 2H, $\left.\mathrm{CH}_{2} \mathrm{NH}\right), 4.53$ (s, 2H, $\left.\underline{\mathrm{C}}_{2} \mathrm{Ph}\right), 7.32-7.75$ (m, 9H, Har.), $8.91(\mathrm{~s}, 1 \mathrm{H}, \mathrm{NH})$ ppm. ${ }^{13} \mathbf{C}-\mathbf{N M R}$ (DMSO-d $)=$ $\delta 21.25\left(\mathrm{CH}_{3}\right), 42.41\left(\mathrm{CH}_{2}\right), 154.13,159.73,163.93$, 127.07, 130.06, 137.08, 143.73, $148.65\left(\mathrm{C}_{\mathrm{ar} .}\right), 158.92$ $(\mathrm{C}=\mathrm{N}), 170.02\left(\mathrm{C}=\mathrm{O}_{\text {cyclic }}\right) \mathrm{ppm}$.

N-(1-(3-Benzyl-4-oxo-4,8a-dihydro-1H-[1,3,4]thiadiazolo[2,3-c][1,2,4]triazin-7-yl)ethyl)-4-met-hylbenzene sulfonamide $(7 \mathbf{k})$.

IR $(\mathrm{KBr}) v\left(\mathrm{~cm}^{-1}\right)=1332,1371\left(\mathrm{SO}_{2}\right), 1563(\mathrm{C}=\mathrm{N}), 1715$ $(\mathrm{C}=\mathrm{O}), 2995(\mathrm{CH}), 3107\left(\mathrm{CH}_{\mathrm{ar}}\right), 3395(\mathrm{NH}) .{ }^{\mathbf{1}} \mathbf{H}-\mathbf{N M R}$ $\left(\mathrm{DMSO}-d_{6}\right)=\delta 2.31\left(\mathrm{~s}, 3 \mathrm{H}, \mathrm{CH}_{3} \mathrm{CH}\right), 2.52(\mathrm{~s}, 3 \mathrm{H}$, $\left.\mathrm{CH}_{3} \mathrm{Ph}\right), 3.22(\mathrm{~s}, 1 \mathrm{H}, \mathrm{CH}), 3.45\left(\mathrm{~s}, 2 \mathrm{H}, \mathrm{CH}_{2}\right), 7.32-7.75$ (m, 9H, Har.), 7.95 (s, 1H, NH) ppm. ${ }^{13} \mathbf{C}-\mathbf{N M R}$ (DMSO$\left.d_{6}\right)=\delta 17.30\left(\mathrm{CH}_{3}\right), 26.13\left(\underline{\mathrm{CH}}_{3} \mathrm{CH}\right), 39.35\left(\mathrm{CH}_{2}\right), 154.46$, $160.81,162.56,127.51,129.54,138.14,143.52,149.34$ $\left(\mathrm{C}_{\mathrm{ar}}\right), 159.31(\mathrm{C}=\mathrm{N}), 169.62\left(\mathrm{C}=\mathrm{O}_{\text {cyclic }}\right) \mathrm{ppm}$.

N-(1-(3-Benzyl-4-oxo-4,8a-dihydro-1 $H$ - $[1,3,4]$ thiadiazolo[2,3-c][1,2,4]triazin-7-yl)-2-phenyleth-yl)-4methylbenzenesulfonamide (7I).

IR $(\mathrm{KBr}) v\left(\mathrm{~cm}^{-1}\right)=1338,1368\left(\mathrm{SO}_{2}\right), 1646(\mathrm{C}=\mathrm{N}), 1718$ $(\mathrm{C}=\mathrm{O}), 2851\left(\mathrm{CH}_{2}\right), 3063\left(\mathrm{CH}_{\mathrm{ar}}\right), 3352(\mathrm{NH}) .{ }^{\mathbf{1}} \mathbf{H}-\mathbf{N M R}$ $\left(\mathrm{DMSO}-d_{6}\right)=\delta 1.31\left(\mathrm{~s}, 3 \mathrm{H}, \mathrm{CH}_{3} \mathrm{CH}\right), 2.53(\mathrm{~s}, 2 \mathrm{H}$, $\left.\mathrm{CH}_{2} \mathrm{CH}\right), 4.01$ (s, $\left.1 \mathrm{H}, \mathrm{CH}\right), 4.20\left(\mathrm{~s}, 2 \mathrm{H}, \mathrm{CH}_{2} \mathrm{Ph}\right), 7.41-7.90$ (m, 14H, Har.), 8.55 (s, 1H, NH) ppm. ${ }^{13} \mathbf{C}-\mathbf{N M R}$ (DMSO$\left.d_{6}\right)=\delta 14.17\left(\mathrm{CH}_{3}\right), 39.29\left(\mathrm{CH}_{2}\right), 61.12(\mathrm{CH}), 126.59$, $127.44,129.00,129.96,130.15,130.50,135.50$ and $139.93\left(\mathrm{C}_{\text {ar. }}\right), 156.30(\mathrm{C}=\mathrm{N}), 168.20\left(\mathrm{C}=\mathrm{O}_{\text {cyclic }}\right) \mathrm{ppm}$.

Table 3: Physical and analytical data of compounds 7a-l.

\begin{tabular}{|l|l|l|l|l|l|l|}
\hline Cpd & $\begin{array}{l}\text { M.P. } \\
\left({ }^{\circ} \mathrm{C}\right)\end{array}$ & $\begin{array}{l}\text { Yield } \\
(\%)\end{array}$ & M. F. & \multicolumn{4}{|l|}{ M.A. ( \%); } \\
(M. W. $)$ & \multicolumn{3}{|l|}{ Calcd/Found } \\
$\mathrm{C} \% \quad \mathrm{H} \%$ & $\mathrm{~N} \%$ \\
\hline $\mathbf{7 a}$ & $176-$ & 83 & $\mathrm{C}_{19} \mathrm{H}_{17} \mathrm{~N}_{5} \mathrm{O}_{3} \mathrm{~S}_{2}$ & 53.38 & 4.01 & 16.38 \\
\hline
\end{tabular}

Delta J. Sci. 2016 ; Vol. 38 : (0-0)

\begin{tabular}{|c|c|c|c|c|c|c|}
\hline & 8 & & $(427.5)$ & 53.09 & 3.92 & 15.98 \\
\hline \multirow{2}{*}{$7 b$} & \multirow{2}{*}{$\begin{array}{l}183- \\
5\end{array}$} & \multirow{2}{*}{71} & \multirow{2}{*}{$\begin{array}{l}\mathrm{C}_{20} \mathrm{H}_{19} \mathrm{~N}_{5} \mathrm{O}_{3} \mathrm{~S}_{2} \\
(441.53)\end{array}$} & 54.41 & 4.34 & 15.86 \\
\hline & & & & 54.12 & 3.97 & 15.57 \\
\hline \multirow{2}{*}{$7 c$} & \multirow{2}{*}{$\begin{array}{l}179- \\
81\end{array}$} & \multirow{2}{*}{78} & $\mathrm{C}_{26} \mathrm{H}_{22} \mathrm{~N}_{5} \mathrm{O}_{3} \mathrm{~S}_{2}$ & 60.45 & 4.29 & 13.56 \\
\hline & & & $(516.61)$ & 60.15 & 3.07 & 13.27 \\
\hline \multirow{2}{*}{$7 d$} & \multirow{2}{*}{$\begin{array}{l}197- \\
9\end{array}$} & \multirow{2}{*}{85} & \multirow{2}{*}{$\begin{array}{l}\mathrm{C}_{20} \mathrm{H}_{19} \mathrm{~N}_{5} \mathrm{O}_{3} \mathrm{~S}_{2} \\
(441.53)\end{array}$} & 54.41 & 4.34 & 15.86 \\
\hline & & & & 54.22 & 3.97 & 15.45 \\
\hline \multirow{2}{*}{$7 e$} & \multirow{2}{*}{$\begin{array}{l}158- \\
60\end{array}$} & \multirow{2}{*}{69} & \multirow{2}{*}{$\begin{array}{l}\mathrm{C}_{21} \mathrm{H}_{21} \mathrm{~N}_{5} \mathrm{O}_{3} \mathrm{~S}_{2} \\
(455.55)\end{array}$} & 55.37 & 4.65 & 15.37 \\
\hline & & & & 55.18 & 4.24 & 15.08 \\
\hline \multirow[b]{2}{*}{$7 f$} & \multirow{2}{*}{$\begin{array}{l}202- \\
4\end{array}$} & \multirow[b]{2}{*}{74} & \multirow{2}{*}{$\begin{array}{l}\mathrm{C}_{27} \mathrm{H}_{25} \mathrm{~N}_{5} \mathrm{O}_{3} \mathrm{~S}_{2} \\
(531.65)\end{array}$} & 61.00 & 4.74 & 13.17 \\
\hline & & & & $\begin{array}{l}60 . \\
14\end{array}$ & 4.38 & 12.98 \\
\hline \multirow{2}{*}{$7 \mathrm{~g}$} & \multirow{2}{*}{$\begin{array}{l}232- \\
4\end{array}$} & \multirow{2}{*}{58} & \multirow{2}{*}{$\begin{array}{l}\mathrm{C}_{18} \mathrm{H}_{17} \mathrm{~N}_{5} \mathrm{O}_{3} \mathrm{~S}_{2} \\
(415.49)\end{array}$} & & 4.12 & 16.86 \\
\hline & & & & 51.81 & 3.93 & 16.57 \\
\hline \multirow{2}{*}{$7 \mathrm{~h}$} & \multirow{2}{*}{$\begin{array}{l}165- \\
7\end{array}$} & \multirow{2}{*}{67} & \multirow{2}{*}{$\begin{array}{l}\mathrm{C}_{19} \mathrm{H}_{19} \mathrm{~N}_{5} \mathrm{O}_{3} \mathrm{~S}_{2} \\
(429.52)\end{array}$} & 53.13 & 4.46 & 16.31 \\
\hline & & & & 52.95 & 4.17 & 16.10 \\
\hline \multirow{2}{*}{$7 \mathbf{i}$} & \multirow{2}{*}{$\begin{array}{l}191- \\
3\end{array}$} & \multirow{2}{*}{79} & $\mathrm{C}_{25} \mathrm{H}_{22} \mathrm{~N}_{5} \mathrm{O}_{3} \mathrm{~S}_{2}$ & 59.51 & 4.39 & 13.88 \\
\hline & & & (504.6) & 59.32 & 4.08 & 13.59 \\
\hline \multirow{2}{*}{$7 \mathbf{j}$} & \multirow{2}{*}{$\begin{array}{l}218- \\
20\end{array}$} & \multirow{2}{*}{61} & \multirow{2}{*}{$\begin{array}{l}\mathrm{C}_{19} \mathrm{H}_{19} \mathrm{~N}_{5} \mathrm{O}_{3} \mathrm{~S}_{2} \\
(429.52)\end{array}$} & 53.13 & 4.46 & 16.31 \\
\hline & & & & 52.83 & 4.27 & 15.92 \\
\hline $7 \mathrm{k}$ & $189-$ & 82 & $\mathrm{C}_{20} \mathrm{H}_{21} \mathrm{~N}_{5} \mathrm{O}_{3} \mathrm{~S}_{2}$ & 54.16 & 4.77 & 15.79 \\
\hline $\mathbf{~} \mathrm{K}$ & 91 & 02 & (443.54) & 53.96 & 4.35 & 15.41 \\
\hline 7 & 199- & 80 & $\mathrm{C}_{26} \mathrm{H}_{25} \mathrm{~N}_{5} \mathrm{O}_{3} \mathrm{~S}_{2}$ & 60.10 & 4.85 & 13.48 \\
\hline 11 & 01 & 87 & (519.64) & 59.97 & 4.58 & 13.17 \\
\hline
\end{tabular}

Hydrolysis of compounds (7a,b and k). Formation of

\section{8a-c.}

Compounds (7a,b and k) $(0.003 \mathrm{~mol})$ was refluxed in conc. hydrochloric $\operatorname{acid}^{13}(25 \mathrm{ml})$ for $3-5 \mathrm{hr}$ (tlc). The reaction mixture was cooled to $\mathrm{r}$. t. and neutralized with sodium hydroxide solution $(10 \%, 25-30 \mathrm{ml})$. The precipitate formed was filtered off, dried, and recrystallized from methanol to form 8a-c. The filtrate was acidified by dil. $\mathrm{HCl}$ to give a white precipitate of 4toluensulphonic acid.

7-(Aminomethyl)-3-styryl-1H-[1,3,4]thiadiazolo-2,3c] $[1,2,4]$ triazin-4(H)-one $(8 a)$.

IR $(\mathrm{KBr}) v\left(\mathrm{~cm}^{-1}\right)=1510(\mathrm{C}=\mathrm{N}), 1623(\mathrm{C}=\mathrm{O}$, cylic $), 2781$ (CH), $3368\left(\mathrm{NH}_{2}\right.$ sym $), 3475\left(\mathrm{NH}_{2}\right.$ asym). ${ }^{1} \mathbf{H}-\mathbf{N M R}$ (DMSO$\left.d_{6}\right)=\delta 2.35\left(\mathrm{~s}, 2 \mathrm{H}, \mathrm{NH}_{2}\right), 3.40\left(\mathrm{~s}, 2 \mathrm{H}, \mathrm{CH}_{2}\right), 4.21-7.35(\mathrm{~d}$, $2 \mathrm{H}, J=2.97 \mathrm{~Hz}, \mathrm{CH}=\mathrm{CH}), 7.65-8.52\left(\mathrm{~m}, 5 \mathrm{H}, \mathrm{H}_{\text {ar. }}\right), 7.95$ $(\mathrm{s}, 1 \mathrm{H}, \mathrm{NH}) \mathrm{ppm} . \quad{ }^{13} \mathbf{C}-\mathbf{N M R}\left(\mathrm{DMSO}-d_{6}\right)=\delta 31.03$ $\left(\mathrm{CH}_{2}\right), 39.70(\mathrm{CH}=\mathrm{CH}), 126.50,126.65,126.84,129.76$, 137.14 and $137.25\left(\mathrm{C}_{\mathrm{ar}}\right), 156.12(\mathrm{C}=\mathrm{N}), 167.68(\mathrm{C}=\mathrm{O})$ ppm.

7-(1-Aminoethyl)-3-styryl-1H-[1,3,4] thiadiazolo-[2,3c] $[1,2,4]$ triazin-4(H)-one $(8 b)$. 
IR $(\mathrm{KBr}) v\left(\mathrm{~cm}^{-1}\right)=1585(\mathrm{C}=\mathrm{N}), 1630(\mathrm{C}=\mathrm{O}$, cylic $), 2818$ $(\mathrm{CH}), 3228\left(\mathrm{NH}_{2}\right.$ sym $), 3398\left(\mathrm{NH}_{2}\right.$ asym $) .{ }^{1} \mathbf{H}-\mathbf{N M R}$ (DMSO$\left.d_{6}\right)=\delta 2.23\left(\mathrm{~s}, 3 \mathrm{H}, \mathrm{CH}_{3}\right), 2.55(\mathrm{~s}, 1 \mathrm{H}, \mathrm{CH}), 4.34(\mathrm{~s}, 2 \mathrm{H}$, $\left.\mathrm{NH}_{2}\right), 4.62-7.43(\mathrm{~d}, 2 \mathrm{H}, J=3.41 \mathrm{~Hz}, \mathrm{CH}=\mathrm{CH}), 7.6-8.45$ $\left(\mathrm{m}, 5 \mathrm{H}, \mathrm{H}_{\text {ar. }}\right), 7.95$ (s, 1H, NH) ppm. ${ }^{13} \mathbf{C}-\mathbf{N M R}$ (DMSO$\left.d_{6}\right)=\delta 21.01\left(\mathrm{CH}_{3}\right), 39.70$ and $39.91(\mathrm{CH}=\mathrm{CH}), 110.81$, $126.43,126.62,128.91,129.67,134.20$ and $138.09\left(\mathrm{C}_{\mathrm{ar}}\right)$, $158.12(\mathrm{C}=\mathrm{N}), 169.32(\mathrm{C}=\mathrm{O}) \mathrm{ppm}$.

7-(1-Aminoethyl)-3-benzyl-1H-[1,3,4]thiadiazo-lo[2,3c] $[1,2,4]$ triazin-4(H)-one $(8 \mathrm{c})$.

IR $(\mathrm{KBr}) v\left(\mathrm{~cm}^{-1}\right)=1600(\mathrm{C}=\mathrm{N}), 1680\left(\mathrm{C}=\mathrm{O}_{\text {cylic }}\right), 3105$ $\left(\mathrm{CH}_{\mathrm{ar}}\right), 3322\left(\mathrm{NH}_{2}\right) .{ }^{1} \mathbf{H}-\mathrm{NMR}$ (DMSO-d $)=\delta 1.03(\mathrm{~s}$, $\left.3 \mathrm{H}, \mathrm{CH}_{3}\right), 2.48(\mathrm{~s}, \mathrm{H}, \mathrm{CH}), 3.49\left(\mathrm{~s}, 2 \mathrm{H}, \mathrm{CH}_{2}\right), 4.18(\mathrm{~s}, 2 \mathrm{H}$, $\left.\mathrm{NH}_{2}\right), 7.46-8.52\left(\mathrm{~m}, 5 \mathrm{H}, \mathrm{H}_{\text {ar. }}\right)$ ppm. ${ }^{13} \mathbf{C}-\mathbf{N M R}$ (DMSO$\left.d_{6}\right)=\delta 12.81\left(\mathrm{CH}_{3}\right), 20.95\left(\mathrm{CH}_{2}\right), 39.82(\mathrm{CH}), 124.32$, $127.15,127.95,129.29,137.18$ and $139.95\left(\mathrm{C}_{\text {ar. }}\right), 158.19$ $(\mathrm{C}=\mathrm{N}), 169.99(\mathrm{C}=\mathrm{O}) \mathrm{ppm}$.

Table 4: Physical and analytical data of compounds 8a-c

\begin{tabular}{|c|c|c|c|c|c|c|}
\hline Cpd & $\begin{array}{l}\text { M.P. } \\
\left({ }^{\circ} \mathrm{C}\right)\end{array}$ & $\begin{array}{l}\text { Yield } \\
(\%)\end{array}$ & $\begin{array}{l}\text { M. F. } \\
\text { (M. W.) }\end{array}$ & $\begin{array}{l}\text { M.A. } \\
\text { Calcd/ } \\
\mathrm{C} \%\end{array}$ & $\begin{array}{l}\text { o); } \\
\text { bund } \\
\mathrm{H} \%\end{array}$ & N\% \\
\hline $8 a$ & $195-7$ & 81 & $\begin{array}{l}\mathrm{C}_{13} \mathrm{H}_{13} \mathrm{~N}_{5} \mathrm{OS} \\
(287.34)\end{array}$ & $\begin{array}{l}54.34 \\
53.99\end{array}$ & $\begin{array}{l}4.56 \\
4.32\end{array}$ & $\begin{array}{l}24.37 \\
24.01\end{array}$ \\
\hline $8 b$ & $187-9$ & 75 & $\begin{array}{l}\mathrm{C}_{14} \mathrm{H}_{15} \mathrm{~N}_{5} \mathrm{OS} \\
(301.37)\end{array}$ & $\begin{array}{l}55.80 \\
55.54\end{array}$ & $\begin{array}{l}5.02 \\
4.72\end{array}$ & $\begin{array}{l}23.24 \\
22.81\end{array}$ \\
\hline $8 c$ & $182-4$ & 78 & $\begin{array}{l}\mathrm{C}_{13} \mathrm{H}_{15} \mathrm{~N}_{5} \mathrm{OS} \\
(289.36)\end{array}$ & $\begin{array}{l}53.96 \\
53.71\end{array}$ & $\begin{array}{l}5.23 \\
5.05\end{array}$ & $\begin{array}{l}24.20 \\
23.97\end{array}$ \\
\hline
\end{tabular}

Reaction of 3a,b with 2-substituted-1,3-benzoxazin-4Hone derivatives. Formation of 10a-d.

A mixture of 3a, b $(0.01 \mathrm{~mol})$ and 2-(phenyl and/or ethoxy)-1,3-benzoxazin-4H-ones $(\mathbf{9 a}, \mathbf{b})(0.01 \mathrm{~mol})$ was refluxed in glacial acetic acid $(20 \mathrm{ml})$ for $13-15 \mathrm{hr}$ (tlc). The solvent was evaporated till dryness and the solid obtained was recrystallized from (methanol / water) to afford 10a-d.

3-(6-Benzyl-5-oxo-3-thioxo-2,3-dihydro-1,2,4-triazin4(5H)-yl)-2-phenylquina-zolin-4(3H)-one (10a).

IR $(\mathrm{KBr}) v\left(\mathrm{~cm}^{-1}\right)=1270(\mathrm{C}=\mathrm{S}), 1600(\mathrm{C}=\mathrm{N}), 1695,1730$ $(2 \mathrm{C}=\mathrm{O}), 2985\left(\mathrm{CH}_{\text {aliph. }}\right), 3088\left(\mathrm{CH}_{\text {ar. }}\right), 3241(\mathrm{NH}) .{ }^{\mathbf{1}} \mathbf{H}-$ NMR $\left(\mathrm{DMSO}-d_{6}\right)=\delta 2.18\left(\mathrm{~s}, 2 \mathrm{H}, \mathrm{CH}_{2}\right), 7.20-8.55(\mathrm{~m}$, $\left.14 \mathrm{H}, \mathrm{H}_{\mathrm{ar}}\right), 8.6(\mathrm{~s}, 1 \mathrm{H}, \mathrm{NH}) \mathrm{ppm} .{ }^{13} \mathbf{C}-\mathbf{N M R}\left(\mathrm{DMSO}-d_{6}\right)=$ $\delta 23.00\left(\mathrm{CH}_{2}\right), 115.58,115.9,125.20,127.90,127.95$, 128.51, 129.06 and $129.19\left(\mathrm{C}_{\mathrm{ar}}\right), 131.70\left(\mathrm{C}-6^{\prime}\right), 131.76$ (C-2'), 135.23 (C-4'), 135.31(C-5'), 135.73 (C-3'), 162.01 $(\mathrm{C}=\mathrm{N}), 172.81(\mathrm{C}=\mathrm{O}), 189.77(\mathrm{C}=\mathrm{S}) \mathrm{ppm}$.

3-(6-Benzyl-5-oxo-3-thioxo-2,3-dihydro-1,2,4-triazin4(5H)-yl)-2-ethoxyquina-zolin-4(3H)-one (10b).

IR $(\mathrm{KBr}) v\left(\mathrm{~cm}^{-1}\right)=1180(\mathrm{C}=\mathrm{S}), 1657(\mathrm{C}=\mathrm{N}), 1725$ $(\mathrm{C}=\mathrm{O}), 2845(\mathrm{CH}), 3085\left(\mathrm{CH}_{\mathrm{ar} .}\right), 3375(\mathrm{NH}) .{ }^{13} \mathbf{C}-\mathbf{N M R}$

Delta J. Sci. 2016 ; Vol. 38 : (0-0)
$\left(\mathrm{DMSO}-d_{6}\right)=\delta 14.17\left(\mathrm{CH}_{3}\right), 39.29\left(\mathrm{CH}_{2}\right), 126.21,128.20$, 128.77, 128.93, 129.90, 130.06, 133.47 and $135.28\left(\mathrm{C}_{\mathrm{ar} .}\right)$, $168.24(\mathrm{C}=\mathrm{N}), 188.02(\mathrm{C}=\mathrm{O}), 193.50(\mathrm{C}=\mathrm{S}) \mathrm{ppm}$.

3-(5-Oxo-6-styryl-3-thioxo-2,3-dihydro-1,2,4-triazin4(5H)-yl)-2-phenyl-quinazolin-4(3H)-one $(10 \mathrm{c})$.

IR $(\mathrm{KBr}) v\left(\mathrm{~cm}^{-1}\right)=1165(\mathrm{C}=\mathrm{S}), 1600(\mathrm{C}=\mathrm{N}), 1718$ $(\mathrm{C}=\mathrm{O}), 2953(\mathrm{CH}), 3105\left(\mathrm{CH}_{\mathrm{ar}}\right), 3380(\mathrm{NH}) .{ }^{\mathbf{1}} \mathbf{H}-\mathbf{N M R}$ $\left(\mathrm{DMSO}-d_{6}\right)=\delta$ 3.72-7.25 $(\mathrm{d}, 2 \mathrm{H}, J=3.32 \mathrm{~Hz}, \mathrm{CH}=\mathrm{CH})$, $7.5-8.20\left(\mathrm{~m}, 14 \mathrm{H}, \mathrm{H}_{\mathrm{ar}}\right), 8.51(\mathrm{~s}, 1 \mathrm{H}, \mathrm{NH}) \mathrm{ppm}$.

2-Ethoxy-3-(5-oxo-6-styryl-3-thioxo-2,3-dihydro-1,2,4triazin-4(5H)-yl)-quinazolin-4(3H)-one (10d).

IR $(\mathrm{KBr}) \cup\left(\mathrm{cm}^{-1}\right)=1155(\mathrm{C}=\mathrm{S}), 1525(\mathrm{C}=\mathrm{N}), 1700$ $(\mathrm{C}=\mathrm{O}), 2910(\mathrm{CH}), 3012\left(\mathrm{CH}_{\mathrm{ar}}\right), 3274(\mathrm{NH})$.

Table 5: Physical and analytical data of compounds 10a-d

\begin{tabular}{|c|c|c|c|c|c|c|}
\hline Cpd & $\begin{array}{l}\text { M.p. } \\
\left({ }^{\circ} \mathrm{C}\right)\end{array}$ & $\begin{array}{l}\text { Yield } \\
(\%)\end{array}$ & $\begin{array}{l}\text { M. F. } \\
\text { (M. W.) }\end{array}$ & \multicolumn{2}{|c|}{$\begin{array}{l}\text { M.A. ( \%); } \\
\text { Calcd/Found } \\
\text { C\% } \\
\text { N\% }\end{array}$} & $\mathrm{H} \%$ \\
\hline $10 \mathrm{a}$ & $\begin{array}{l}190- \\
92\end{array}$ & 55 & $\begin{array}{l}\mathrm{C}_{25} \mathrm{H}_{17} \mathrm{~N}_{5} \mathrm{O}_{2} \mathrm{~S} \\
(451.5)\end{array}$ & $\begin{array}{l}66.50 \\
66.18\end{array}$ & $\begin{array}{l}3.80 \\
3.71\end{array}$ & $\begin{array}{l}15.51 \\
15.43\end{array}$ \\
\hline $10 \mathrm{~b}$ & $\begin{array}{l}186- \\
8\end{array}$ & 68 & $\begin{array}{l}\mathrm{C}_{21} \mathrm{H}_{17} \mathrm{~N}_{5} \mathrm{O}_{3} \mathrm{~S} \\
(419.46)\end{array}$ & $\begin{array}{l}60.13 \\
60.01\end{array}$ & $\begin{array}{l}4.09 \\
3.80\end{array}$ & $\begin{array}{l}16.70 \\
16.59\end{array}$ \\
\hline $10 \mathrm{c}$ & $\begin{array}{l}157- \\
9\end{array}$ & 71 & $\begin{array}{l}\mathrm{C}_{24} \mathrm{H}_{17} \mathrm{~N}_{5} \mathrm{O}_{2} \mathrm{~S} \\
(439.49)\end{array}$ & $\begin{array}{l}65.59 \\
65.36\end{array}$ & $\begin{array}{l}3.90 \\
3.78\end{array}$ & $\begin{array}{l}15.94 \\
15.85\end{array}$ \\
\hline $10 d$ & $\begin{array}{l}198- \\
200\end{array}$ & 57 & $\begin{array}{l}\mathrm{C}_{20} \mathrm{H}_{17} \mathrm{~N}_{5} \mathrm{O}_{3} \mathrm{~S} \\
(407.45)\end{array}$ & $\begin{array}{l}58.96 \\
58.75\end{array}$ & $\begin{array}{l}4.21 \\
3.92\end{array}$ & $\begin{array}{l}17.19 \\
16.95\end{array}$ \\
\hline
\end{tabular}

Reaction of 4-amino-6-benzyl-3-thioxo-3,4-dihydro-

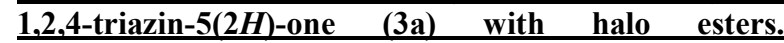
Formation of 12 and 13.

Compound 3a (2.34 g., $0.01 \mathrm{~mol})$ was fused with ethyl chloro -(acetate and / or acetoacetate) $\left(10 \mathrm{ml}\right.$.) at $170{ }^{\circ} \mathrm{C}$ or refluxed in methanol in the presence of sodium hydroxide for 3-4 hr (tlc). The excess solvent was evaporated till dryness. The residual solid was crystallized from petroleum ether $\left(80-100^{\circ} \mathrm{C}\right)$ and filtered to give $\mathbf{1 2}$ and $\mathbf{1 3}$, respectively.

Ethyl-2-(4-amino-6-benzyl-5-oxo-2,3,4,5-tetrahydro1,2,4-triazin-3-ylthio)- acetate (12).

IR $(\mathrm{KBr}) \vee\left(\mathrm{cm}^{-1}\right)=1631(\mathrm{C}=\mathrm{N}), 1713,1684(2 \mathrm{C}=\mathrm{O})$, $2954\left(\mathrm{CH}_{2}\right), 3146\left(\mathrm{CH}_{\mathrm{ar}}\right), 3452\left(\mathrm{NH}_{2} \mathrm{sym}\right), 3555\left(\mathrm{NH}_{2}\right.$ asym.). ${ }^{1} \mathbf{H}-\mathrm{NMR}$ (DMSO- $\left.d_{6}\right) \delta=1.23(\mathrm{t}, 3 \mathrm{H}, J=3.62 \mathrm{~Hz}$, $\left.\mathrm{CH}_{3}\right), 2.50$ (s, $\left.2 \mathrm{H}, \mathrm{NH}_{2}\right), 3.30\left(\mathrm{~s}, 2 \mathrm{H}, \mathrm{CH}_{2} \mathrm{~S}\right), 3.55(\mathrm{~s}, 2 \mathrm{H}$, $\mathrm{CH}_{2} \mathrm{Ph}$ ), 4.3 (q, $\left.2 \mathrm{H}, J=2.57 \mathrm{~Hz}, \mathrm{CH}_{2} \mathrm{CH}_{3}\right), 6.90-8.21$ $\left(\mathrm{m}, 5 \mathrm{H}, \mathrm{H}_{\mathrm{ar}}\right) \mathrm{ppm}$.

Ethyl-2-(4-amino-6-benzyl-5-oxo-2,3,4,5-tetrahyd-ro1,2,4-triazin-3-ylthio)-3-oxo-butanoate (13).

IR $(\mathrm{KBr})$ v $\left(\mathrm{cm}^{-1}\right)=1571(\mathrm{C}=\mathrm{N}), 1619(\mathrm{C}=\mathrm{O}), 2912$ $\left(\mathrm{CH}_{2}\right), 3001$ ( $\left.\mathrm{CH}_{\text {ar. }}\right), 3338\left(\mathrm{NH}_{2}\right.$ sym. $), 3453\left(\mathrm{NH}_{2}\right.$ asym. $) .{ }^{1} \mathbf{H}-$ 
NMR $\left(\right.$ DMSO- $\left.d_{6}\right) \delta=1.25\left(\mathrm{t}, 3 \mathrm{H}, J=3.42 \mathrm{~Hz}, \mathrm{CH}_{3} \mathrm{CH}_{2}\right)$, $1.65\left(\mathrm{~s}, 3 \mathrm{H}, \mathrm{CH}_{3} \mathrm{CO}\right), 2.5\left(\mathrm{~s}, 2 \mathrm{H}, \mathrm{NH}_{2}\right), 3.2(\mathrm{~s}, 2 \mathrm{H}$, $\mathrm{CH}_{2} \mathrm{Ph}$ ), 3.4 (q, 2H, $\left.J=2.51 \mathrm{~Hz}, \mathrm{CH}_{2} \mathrm{CH}_{3}\right), 3.55(\mathrm{~s}, 1 \mathrm{H}$, $\mathrm{CH}), 7.11-8.5\left(\mathrm{~m}, 5 \mathrm{H}, \mathrm{H}_{\mathrm{ar}}\right) \mathrm{ppm}$.

Table 6. Physical and analytical data of compounds 12 and 13.

\begin{tabular}{|c|c|c|c|c|c|c|}
\hline \multirow{3}{*}{$\begin{array}{l}\text { Cpd } \\
\mathbf{1 2}\end{array}$} & \multirow{2}{*}{$\begin{array}{l}\text { M.P } \\
\left({ }^{\circ} \mathrm{C}\right)\end{array}$} & \multirow{2}{*}{$\begin{array}{l}\text { Yiel } \\
d \\
(\%)\end{array}$} & \multirow{2}{*}{$\begin{array}{l}\text { M.F. } \\
\text { (M. wt.) }\end{array}$} & \multicolumn{3}{|c|}{$\begin{array}{l}\text { M.A. ( } \%) ; \\
\text { Calcd/Found }\end{array}$} \\
\hline & & & & $\begin{array}{l}\mathrm{C} \% \\
\mathrm{~N} \%\end{array}$ & & $\mathrm{H} \%$ \\
\hline & $\begin{array}{c}178 \\
-80\end{array}$ & 74 & $\begin{array}{l}\mathrm{C}_{14} \mathrm{H}_{16} \mathrm{~N}_{4} \mathrm{O}_{3} \\
\mathrm{~S}(320.37)\end{array}$ & $\begin{array}{l}52.4 \\
9 \\
52.3 \\
7\end{array}$ & $\begin{array}{l}5.0 \\
3 \\
4.6 \\
2\end{array}$ & $\begin{array}{l}17.4 \\
9 \\
17.3 \\
8\end{array}$ \\
\hline 13 & $\begin{array}{l}205 \\
-7\end{array}$ & 83 & $\begin{array}{l}\mathrm{C}_{16} \mathrm{H}_{18} \mathrm{~N}_{4} \mathrm{O}_{4} \\
\mathrm{~S} \\
(362.4)\end{array}$ & $\begin{array}{l}53.0 \\
3 \\
52.8 \\
4\end{array}$ & $\begin{array}{l}5.0 \\
1 \\
4.7 \\
2\end{array}$ & $\begin{array}{l}15.4 \\
6 \\
15.3 \\
7\end{array}$ \\
\hline
\end{tabular}

dioxoisoindolealkyl acetic acid derivatives. Formation of 15a-f.

A mixture of 3a,b $(0.001 \mathrm{~mol})$ and (1,3-dioxo-1,3dihydroisoindol-2-yl)(methyl-, phenyl- and/or benzyl)acetic acid ( 14a-f) $(0.001 \mathrm{~mol})$ (prepared by fusion of glycine, alanine and/or phenyl alanine with phthalic anhydride according to Okuda et al $)^{13-15}$ was heated in phosphorus oxychloride $(20 \mathrm{ml})$ at $85{ }^{\circ} \mathrm{C}$ for $5-6 \mathrm{hr}(\mathrm{tlc})$. The reaction mixture was evaporated till dryness, cooled and neutralized with sodium hydroxide solution $(10 \%, 30$ $\mathrm{ml})$. The precipitate formed was filtered off, dried, and recrystallized from methanol and yielded 15a-f.

2-((4-Oxo-3-styryl-4,8a-dihydro-1H-[1,3,4] thiadiazolo[2,3-c]-[1,2,4]triazin-7-yl)methyl)isoindo-line-1,3dione (15a).

IR $(\mathrm{KBr}) v\left(\mathrm{~cm}^{-1}\right)=1460\left(\mathrm{CH}_{2}\right) 1538\left(\mathrm{C}=\mathrm{C}_{\mathrm{ar}}\right), 1642$ $(\mathrm{C}=\mathrm{N}), 1721(\mathrm{C}=\mathrm{O}, \mathrm{NCO}), 1772(\mathrm{C}=\mathrm{O}, \underline{\mathrm{CONCO}}), 2997$ (CH), $3099\left(\mathrm{CH}_{\mathrm{ar}}\right), 3445(\mathrm{NH}) .{ }^{1} \mathbf{H}-\mathrm{NMR}\left(\mathrm{DMSO}-d_{6}\right) \delta=$ $3.55\left(\mathrm{~s}, 2 \mathrm{H}, \mathrm{CH}_{2}\right), 5.21-5.42(\mathrm{~d}, 1 \mathrm{H}, J=3.12 \mathrm{~Hz}$, $\mathrm{CH}=\mathrm{CH}), 7.82-9.75\left(\mathrm{~m}, 9 \mathrm{H}, \mathrm{H}_{\mathrm{ar}}\right), 10.15(\mathrm{~s}, 1 \mathrm{H}, \mathrm{NH})$ ppm. ${ }^{13} \mathbf{C}-\mathbf{N M R}\left(\mathrm{DMSO}-d_{6}\right) \delta=32.18\left(\mathrm{CH}_{2}\right), 51.65$ $(\mathrm{CH}), 146.52,153.18,124.39,131.59$ and $134.73\left(\mathrm{C}_{\mathrm{ar}}\right)$, $165.61(\mathrm{C}=\mathrm{N}), 175.37(\underline{\mathrm{CON}} \underline{\mathrm{CO}}) \mathrm{ppm}$.

2-(1-(4-Oxo-3-styryl-4,8a-dihydro-1H[1,3,4]thiadiazolo[2,3-c] $[1,2,4]$-triazin-7-yl)ethyl)isoind-oline-1,3dione (15b).

IR $(\mathrm{KBr}) \cup\left(\mathrm{cm}^{-1}\right)=1495\left(\mathrm{C}=\mathrm{C}_{\text {ar. }}\right), 1626(\mathrm{C}=\mathrm{N}), 1714$ $(\mathrm{C}=\mathrm{O}), 2925\left(\mathrm{CH}, \mathrm{CH}_{3}\right), 3028\left(\mathrm{CH}_{\mathrm{ar}}\right), 3425(\mathrm{NH}) .{ }^{1} \mathbf{H}-$ NMR $\left(\mathrm{DMSO}-d_{6}\right) \delta=2.15\left(\mathrm{~s}, 3 \mathrm{H}, \mathrm{CH}_{3}\right), 2.25,(\mathrm{~s}, 1 \mathrm{H}$, $\mathrm{CH}), 4.36-4.63(\mathrm{~d}, 2 \mathrm{H}, J=2.98 \mathrm{~Hz}, \mathrm{CH}=\mathrm{CH}), 7.78-8.03$ $\left(\mathrm{m}, 9 \mathrm{H}, \mathrm{H}_{\text {ar. }}\right)$ ppm. ${ }^{13} \mathbf{C}-\mathbf{N M R}\left(\mathrm{DMSO}-d_{6}\right) \delta=16.75$
$\left(\mathrm{CH}_{3}\right), 52.63(\mathrm{CH}), 125.37,131.69,134.74,148.55$ and $157.27\left(\mathrm{C}_{\mathrm{ar}}\right), 167.61(\mathrm{C}=\mathrm{N}), 174.47(\underline{\mathrm{CONCO}}) \mathrm{ppm}$.

\section{2-(1-(4-Oxo-3-styryl-4,8a-dihydro-1H-[1,3,4-} ]thiadiazolo[2,3-c][1,2,4]-triazin-7-yl)-2-phenylethyl)isoindoline-1,3-dione (15c).

IR $(\mathrm{KBr}) v\left(\mathrm{~cm}^{-1}\right)=1491\left(\mathrm{CH}_{2}\right), 1515\left(\mathrm{C}=\mathrm{C}_{\mathrm{ar}}\right), 1622$ $(\mathrm{C}=\mathrm{N}), 1725(\mathrm{C}=\mathrm{O}), 3020\left(\mathrm{CH}_{\mathrm{ar} .}\right), 3315(\mathrm{NH}) .{ }^{\mathbf{1}} \mathbf{H}-\mathbf{N M R}$ $\left(\mathrm{DMSO}-d_{6}\right) \delta=3.11\left(\mathrm{~s}, 2 \mathrm{H}, \mathrm{CH}_{2}\right), 3.73(\mathrm{~s}, 1 \mathrm{H}, \mathrm{CH}), 7.15-$ $7.35(\mathrm{~d}, 2 \mathrm{H}, J=3.50 \mathrm{~Hz}, \mathrm{CH}=\mathrm{CHPh}), 7.85-10.50(\mathrm{~m}$, $14 \mathrm{H}, \mathrm{H}_{\mathrm{ar}}$ ), 10.92 (s, 1H, NH.) ppm. ${ }^{13} \mathbf{C}-\mathbf{N M R}$ (DMSO-d ${ }_{6}$ ) $\delta=37.45\left(\mathrm{CH}_{2}\right), 61.15(\underline{\mathrm{CH}}), 121.54,124.89,127.59$, $129.14,131.72,135.73148 .78$ and $157.18\left(\mathrm{C}_{\mathrm{ar} .}\right), 161.55$ $(\mathrm{C}=\mathrm{N}), 169.93$ (ㅁN드) ppm.

2-((3-Benzyl-4-oxo-4,8a-dihydro-1H-[1,3,4] thiadiazolo[2,3-c] $[1,2,4]$-triazin-7-yl)-methyl)isoindo-line-1,3dione (15d).

IR $(\mathrm{KBr})$ v $\left(\mathrm{cm}^{-1}\right)=1511\left(\mathrm{CH}_{2}\right), 1562\left(\mathrm{C}=\mathrm{C}_{\text {ar. }}\right), 1595$ $(\mathrm{C}=\mathrm{N}), 1660,1720(2 \mathrm{C}=\mathrm{O}), 2989(\mathrm{CH}), 3015\left(\mathrm{CH}_{\mathrm{ar}}\right)$, $3496(\mathrm{NH}) .{ }^{1} \mathbf{H}-\mathrm{NMR}$ (DMSO-d $d_{6} \delta=2.11(\mathrm{~s}, 2 \mathrm{H}$, $\mathrm{CH}_{2} \mathrm{~N}$ ), 3.63 (s, 2H, $\left.\mathrm{CH}_{2} \mathrm{Ph}\right), 7.25-9.85$ (m, 9H, $\mathrm{H}_{\mathrm{ar}}$ ), 10.47 (s, 1H, NH) ppm. ${ }^{13}$ C-NMR $\left(\right.$ DMSO- $\left._{6}\right) \delta=27.18$ $\left(\mathrm{CH}_{2}\right), 33.39\left(\mathrm{CH}_{2} \mathrm{Ph}\right), 145.47,154.94,125.41,131.19$, $135.71\left(\mathrm{C}_{\mathrm{ar}}\right), 164.45(\mathrm{C}=\mathrm{N}), 174.52$ (ㅁN므) ppm.

2-(1-(3-Benzyl-4-oxo-4,8a-dihydro-1H-[1,3,4]thiadiazolo[2,3-c] $[1,2,4]$ triazin-7-yl) ethyl)iso-indoline-1,3-dione (15e).

IR $(\mathrm{KBr}) \cup\left(\mathrm{cm}^{-1}\right)=1496\left(\mathrm{C}=\mathrm{C}_{\mathrm{ar}}\right), 1571\left(\mathrm{CH}_{2}\right), 1605$ $(\mathrm{C}=\mathrm{N}), 1718,1783(2 \mathrm{C}=\mathrm{O}), 2894\left(\mathrm{CH}, \mathrm{CH}_{3}\right), 3458(\mathrm{NH})$. ${ }^{1} \mathbf{H}-\mathbf{N M R}\left(\mathrm{DMSO}-d_{6}\right) \delta=1.25\left(\mathrm{~s}, 3 \mathrm{H}, \mathrm{CH}_{3}\right), 2.55(\mathrm{~s}, 1 \mathrm{H}$, $\mathrm{CH}), 2.61\left(\mathrm{~s}, 2 \mathrm{H}, \mathrm{CH}_{2}\right), 7.87-9.82\left(\mathrm{~m}, 9 \mathrm{H}, \mathrm{H}_{\mathrm{ar}}\right) \mathrm{ppm}$. ${ }^{13}$ C-NMR (DMSO- $\left.d_{6}\right) \delta=17.81\left(\mathrm{CH}_{3}\right), 28.41\left(\mathrm{CH}_{2}\right)$, $37.01(\mathrm{CH}), 126.41,131.69,135.34,148.05$ and 158.94 $\left(\mathrm{C}_{\mathrm{ar}}\right), 169.22(\mathrm{C}=\mathrm{N}), 177.93$ (ㅁN므) ppm.

2-(1-(3-Benzyl-4-oxo-4,8a-dihydro-1H-[1,3,4]thiadiazolo[2,3-c] $[1,2,4]$ triazin-7-yl)-2-phenylethyl)isoindoline-1,3-dione (15f).

IR $(\mathrm{KBr}) v\left(\mathrm{~cm}^{-1}\right)=1497\left(\mathrm{C}=\mathrm{C}_{\mathrm{ar}}\right), 1555(\mathrm{C}=\mathrm{N}), 1618$ $\left(\mathrm{CH}_{2}\right), 1615,1660(2 \mathrm{C}=\mathrm{O}), 3113\left(\mathrm{CH}_{\mathrm{ar}}\right), 3410(\mathrm{NH}) .{ }^{\mathbf{1}} \mathbf{H}-$ NMR $\left(\mathrm{DMSO}-d_{6}\right) \delta=3.61-3.82\left(\mathrm{~s}, 4 \mathrm{H}, 2 \mathrm{CH}_{2}\right.$ and $\mathrm{H}$, $\mathrm{CH}), 7.8$ - $9.4\left(\mathrm{~m}, 14 \mathrm{H}, \mathrm{H}_{\mathrm{ar}}\right), 9.94(\mathrm{~s}, 1 \mathrm{H}, \mathrm{NH}$ ) $\mathrm{ppm}$. MS (EI) $\mathrm{m} / \mathrm{z}=493.12\left(\mathrm{M}^{+}, \mathrm{C}_{27} \mathrm{H}_{19} \mathrm{~N}_{5} \mathrm{O}_{3} \mathrm{~S}, 1.41 \%\right)$.

Table 7. Physical and analytical data of compounds $\mathbf{1 5}$ a-f.

\begin{tabular}{|c|c|c|c|c|c|c|}
\hline \multirow{3}{*}{$\begin{array}{l}\text { Cpd } \\
. \\
\mathbf{1 5 a}\end{array}$} & \multirow{2}{*}{$\begin{array}{l}\text { M.P } \\
\left({ }^{\circ} \mathrm{C}\right) \\
172\end{array}$} & \multirow{2}{*}{$\begin{array}{l}\text { Yiel } \\
\mathrm{d} \\
(\%) \\
89\end{array}$} & \multirow{2}{*}{$\begin{array}{l}\text { M.F. } \\
\text { (M. wt.) }\end{array}$} & \multicolumn{3}{|c|}{$\begin{array}{l}\text { M.A. ( } \%) ; \\
\text { Calcd/Found }\end{array}$} \\
\hline & & & & $\begin{array}{l}\mathrm{C} \% \\
\mathrm{~N} \%\end{array}$ & & $\mathrm{H} \%$ \\
\hline & $\begin{array}{l}172 \\
-4\end{array}$ & 89 & $\begin{array}{l}\mathrm{C}_{21} \mathrm{H}_{15} \mathrm{~N}_{5} \mathrm{O}_{3} \\
\mathrm{~S}(417.44)\end{array}$ & $\begin{array}{l}60.4 \\
2 \\
60.2\end{array}$ & $\begin{array}{l}3.6 \\
2 \\
3.4\end{array}$ & $\begin{array}{l}16.7 \\
8 \\
16.6\end{array}$ \\
\hline
\end{tabular}




\begin{tabular}{|c|c|c|c|c|c|c|}
\hline & & & & 4 & 5 & 0 \\
\hline 15b & $\begin{array}{l}193 \\
-5\end{array}$ & 52 & $\begin{array}{l}\mathrm{C}_{22} \mathrm{H}_{17} \mathrm{~N}_{5} \mathrm{O}_{3} \\
\mathrm{~S}(431.47)\end{array}$ & $\begin{array}{l}61.2 \\
4 \\
60.9 \\
2\end{array}$ & $\begin{array}{l}3.9 \\
7 \\
3.8 \\
9\end{array}$ & $\begin{array}{l}16.2 \\
3 \\
16.1 \\
2\end{array}$ \\
\hline $15 c$ & $\begin{array}{l}185 \\
-7\end{array}$ & 68 & $\begin{array}{l}\mathrm{C}_{28} \mathrm{H}_{21} \mathrm{~N}_{5} \mathrm{O}_{3} \\
\mathrm{~S}(507.56)\end{array}$ & $\begin{array}{l}66.2 \\
6 \\
66.1 \\
4\end{array}$ & $\begin{array}{l}4.1 \\
7 \\
4.0 \\
3\end{array}$ & $\begin{array}{l}13.8 \\
0 \\
13.7 \\
2\end{array}$ \\
\hline 15d & $\begin{array}{l}205 \\
-7\end{array}$ & 71 & $\begin{array}{l}\mathrm{C}_{20} \mathrm{H}_{15} \mathrm{~N}_{5} \mathrm{O}_{3} \\
\mathrm{~S}(405.43)\end{array}$ & $\begin{array}{l}59.2 \\
5 \\
59.0 \\
8\end{array}$ & $\begin{array}{l}3.7 \\
3 \\
3.6 \\
1\end{array}$ & $\begin{array}{l}17.2 \\
7 \\
17.1 \\
4\end{array}$ \\
\hline 15e & $\begin{array}{l}220 \\
-2\end{array}$ & 86 & $\begin{array}{l}\mathrm{C}_{21} \mathrm{H}_{17} \mathrm{~N}_{5} \mathrm{O}_{3} \\
\mathrm{~S}(419.46)\end{array}$ & $\begin{array}{l}60.1 \\
3 \\
60.0 \\
5\end{array}$ & $\begin{array}{l}4.0 \\
9 \\
3.8 \\
1\end{array}$ & $\begin{array}{l}16.7 \\
0 \\
16.6 \\
4\end{array}$ \\
\hline $15 f$ & $\begin{array}{l}197 \\
-9\end{array}$ & 59 & $\begin{array}{l}\mathrm{C}_{27} \mathrm{H}_{21} \mathrm{~N}_{5} \mathrm{O}_{3} \\
\mathrm{~S}(495.55)\end{array}$ & $\begin{array}{l}65.4 \\
4 \\
65.3 \\
6\end{array}$ & $\begin{array}{l}4.2 \\
7 \\
4.1 \\
2\end{array}$ & $\begin{array}{l}14.1 \\
3 \\
14.0 \\
1\end{array}$ \\
\hline
\end{tabular}

Hydrolysis of compounds 15a,b. Formation of 16a,b.

A mixture of $\mathbf{1 5 a} \mathbf{a}, \mathbf{b}(0.0001 \mathrm{~mol})$ was boiled in conc. $\mathrm{HCl}$ $(25 \mathrm{ml})$ for $8-10 \mathrm{hr}(\mathrm{tlc})$. The reaction mixture was cooled to $r$. t. and neutralized with sodium hydroxide solution (10 $\%, 30 \mathrm{ml}$ ). The precipitate formed was filtered off, dried, and recrystallized from methanol to form $\mathbf{1 6 a}, \mathbf{b}$.

\section{7-(Aminomethyl)-3-styryl-1H-[1,3,4] thiadiazol-o[2,3- c] $[1,2,4]$ triazin-4(8aH)-one (16a).}

IR $(\mathrm{KBr})$ v $\left(\mathrm{cm}^{-1}\right)=1483\left(\mathrm{C}=\mathrm{C}_{\text {ar }}\right), 1514(\mathrm{C}=\mathrm{N}), 1573$ $\left(\mathrm{CH}_{2}\right), 1635(\mathrm{C}=\mathrm{O}), 3075(\mathrm{CH}=\mathrm{CH}), 3368\left(\mathrm{NH}_{2}\right.$ sym $), 3475$ $\left(\mathrm{NH}_{2}\right.$ asym). ${ }^{1} \mathbf{H}-\mathbf{N M R}\left(\mathrm{DMSO}-d_{6}\right) \delta=1.97\left(\mathrm{~s}, 2 \mathrm{H}, \mathrm{CH}_{2}\right)$, $2.21\left(\mathrm{~s}, 2 \mathrm{H}, \mathrm{NH}_{2}\right), 5.55-5.75(\mathrm{~d}, 2 \mathrm{H}, J=3.31 \mathrm{~Hz}$, $\mathrm{CH}=\mathrm{CH}), 6.31-6.46 \quad\left(\mathrm{~m}, 5 \mathrm{H}, \mathrm{H}_{\mathrm{ar}}\right) \mathrm{ppm}$.

\section{7-(1-Aminoethyl)-3-styryl-1H[1,3,4]thiadiazo-lo[2,3- c] $[1,2,4]$ triazin-4(8aH)-one (16b).}

IR $(\mathrm{KBr})$ v $\left(\mathrm{cm}^{-1}\right)=1520\left(\mathrm{C}=\mathrm{C}_{\mathrm{ar}}\right), 1597(\mathrm{C}=\mathrm{N}), 1726$ $(\mathrm{C}=\mathrm{O}), 3072(\mathrm{CH}=\mathrm{CH}), 3310\left(\mathrm{NH}_{2} \mathrm{sym}\right), 3435\left(\mathrm{NH}_{2}\right.$ asym $)$. ${ }^{1} \mathbf{H}-\mathbf{N M R}\left(\mathrm{DMSO}-d_{6}\right) \delta=1.18\left(\mathrm{~s}, 3 \mathrm{H}, \mathrm{CH}_{3}\right), 2.5(\mathrm{~s}, 2 \mathrm{H}$, $\left.\mathrm{NH}_{2}\right), 4.01-6.40(\mathrm{~d}, 2 \mathrm{H}, J=3.11 \mathrm{~Hz}, \mathrm{CH}=\mathrm{CH}), 7.51-8.05$ $\left(\mathrm{m}, 5 \mathrm{H}, \mathrm{H}_{\mathrm{ar}}\right) \mathrm{ppm} .{ }^{13} \mathrm{C}-\mathrm{NMR}\left(\mathrm{DMSO}-d_{6}\right) \delta=14.17\left(\mathrm{CH}_{3}\right)$, $61.12(\mathrm{CH}=\mathrm{CH}), 126.59,127.44,129.00,129.96,130.15$, 135.50 and $139.93\left(\mathrm{C}_{\text {ar. }}\right), 156.30(\mathrm{C}=\mathrm{N}), 168.20(\mathrm{C}=\mathrm{O})$ ppm. MS (EI) m/z $=299.08\left(\mathrm{M}^{+}, \mathrm{C}_{14} \mathrm{H}_{13} \mathrm{~N}_{5} \mathrm{OS}, 100 \%\right)$. Reaction of $3 \mathrm{a}, \mathrm{b}$ with 1,4-butane Sultone. Formation of $\underline{18 a, b}$.

A mixture of 3a,b $(0.001 \mathrm{~mol})$ and 1,4-butanesultone (17) $(0.136 \mathrm{ml}, 0.001 \mathrm{~mol})$ was fused in an oil bath at $140-$ $150{ }^{\circ} \mathrm{C}$ for $6-8 \mathrm{hr}$ (tlc). The reaction mixture was cooled and the produced mass was treated with dil. HCl. The solid formed was filtered off, and recrystallized from ethanol to give $\mathbf{1 8 a}$ and $\mathbf{1 8 b}$, respectively

Table 8. Physical and analytical data of compounds 16a,b.

\begin{tabular}{|c|c|c|c|c|c|c|}
\hline \multirow{3}{*}{$\begin{array}{l}\text { Cpd } \\
\text {. } \\
\mathbf{1 6 a}\end{array}$} & \multirow{3}{*}{$\begin{array}{l}\text { M.P. } \\
\cdot \\
\left({ }^{\circ} \mathrm{C}\right) \\
175- \\
7\end{array}$} & \multirow{3}{*}{$\begin{array}{l}\text { Yiel } \\
d \\
(\%) \\
54\end{array}$} & \multirow{2}{*}{$\begin{array}{l}\text { M.F. } \\
\text { (M. wt.) }\end{array}$} & \multicolumn{3}{|c|}{$\begin{array}{l}\text { M.A. ( \%); } \\
\text { Calcd/Found }\end{array}$} \\
\hline & & & & $\begin{array}{l}\mathrm{C} \% \\
\mathrm{~N} \%\end{array}$ & & $\mathrm{H} \%$ \\
\hline & & & $\begin{array}{l}\mathrm{C}_{13} \mathrm{H}_{13} \mathrm{~N}_{5} \mathrm{O} \\
\mathrm{S}(287.34)\end{array}$ & $\begin{array}{l}54.3 \\
4 \\
54.2 \\
1\end{array}$ & $\begin{array}{l}4.5 \\
6 \\
4.4 \\
8\end{array}$ & $\begin{array}{l}24.3 \\
7 \\
24.1 \\
1\end{array}$ \\
\hline $16 b$ & $\begin{array}{l}197- \\
9\end{array}$ & 61 & $\begin{array}{l}\mathrm{C}_{14} \mathrm{H}_{15} \mathrm{~N}_{5} \mathrm{O} \\
\mathrm{S}(301.37)\end{array}$ & $\begin{array}{l}55.8 \\
0 \\
55.6 \\
3\end{array}$ & $\begin{array}{l}5.0 \\
2 \\
4.9 \\
1\end{array}$ & $\begin{array}{l}23.2 \\
4 \\
23.1 \\
2\end{array}$ \\
\hline
\end{tabular}

6-Benzyl-3-thioxo-3,4-dihydro-1,2,4-triazin-5(2H-)oyl1-butan-1,4-sultam (18a).

IR $(\mathrm{KBr}) v\left(\mathrm{~cm}^{-1}\right)=1170(\mathrm{C}=\mathrm{S}), 1162,1351\left(\mathrm{SO}_{2}\right), 1610$ $(\mathrm{C}=\mathrm{N}), 1665\left(\mathrm{C}=\mathrm{O}\right.$, cyclic), $3472(\mathrm{NH}) .{ }^{13} \mathbf{C}-\mathbf{N M R}(\mathrm{DMSO}-$ $\left.d_{6}\right) \delta=20.89\left(\mathrm{CH}_{2}\right), 22.62\left(\mathrm{CH}_{2}\right), 50.62\left(\mathrm{CH}_{2}\right), 126.43$, 126.66, 128.91, 129.67 and $134.20\left(\mathrm{C}_{\text {ar. }}\right), 150.89(\mathrm{C}=\mathrm{N})$, $168.99(\mathrm{C}=\mathrm{O}), 179.99(\mathrm{C}=\mathrm{S}) \mathrm{ppm}$.

\section{6-Styryl-3-thioxo-3,4-dihydro-1,2,4-triazin-5(2H- )oyl1-butan-1,4-sultam (18b).}

IR $(\mathrm{KBr}) v\left(\mathrm{~cm}^{-1}\right)=1157(\mathrm{C}=\mathrm{S}), 1151,1358\left(\mathrm{SO}_{2}\right), 1600$ $(\mathrm{C}=\mathrm{N}), 1690(\mathrm{C}=\mathrm{O}), 3455(\mathrm{NH}) . \mathbf{M S}(\mathrm{EI}) \mathrm{m} / \mathrm{z}=364.07$ $\left(\mathrm{M}^{+}, \mathrm{C}_{15} \mathrm{H}_{16} \mathrm{~N}_{4} \mathrm{O}_{3} \mathrm{~S}_{2}, 80.12 \%\right)$.

Table 9. Physical and analytical data of compounds 18a,b.

\begin{tabular}{|l|l|l|l|l|l|l|}
\hline \multirow{2}{*}{$\begin{array}{l}\text { Cpd } \\
\cdot\end{array}$} & $\begin{array}{l}\text { M.P. } \\
\text { Yiel }\end{array}$ & $\begin{array}{l}\text { d } \\
\left({ }^{\circ} \mathrm{C}\right)\end{array}$ & $\begin{array}{l}\text { M.F. } \\
(\%)\end{array}$ & $($ M. wt. $)$ & \multicolumn{3}{|l|}{ Calcd/Found } \\
& & & & \multicolumn{3}{|c}{$\mathrm{C} \%$} \\
$\mathrm{~N} \%$ & $\mathrm{H} \%$ \\
\hline $\mathbf{1 8 a}$ & $186-$ & 52 & $\mathrm{C}_{14} \mathrm{H}_{16} \mathrm{~N}_{4} \mathrm{O}_{3}$ & 47.7 & 4.5 & 15.9 \\
& 8 & & $\mathrm{~S}_{2}(352.43)$ & 1 & 8 & 0 \\
& & & 47.4 & 4.4 & 15.7 \\
& & & & 2 & 3 & 1 \\
\hline $\mathbf{1 8 b}$ & $174-$ & 61 & $\mathrm{C}_{15} \mathrm{H}_{16} \mathrm{~N}_{4} \mathrm{O}_{3}$ & 49.4 & 4.4 & 15.3 \\
& 6 & & $\mathrm{~S}_{2}(364.44)$ & 3 & 3 & 7 \\
& & & & 49.3 & 4.3 & 15.1 \\
& & & & 1 & 1 & 9 \\
\hline
\end{tabular}

\section{Biological Activity}

\section{Experimental}

$10 \mathrm{~g}$ peptone, $20 \mathrm{~g}$ glucose, and $20 \mathrm{~g}$ agar were dissolved in $1 \mathrm{~L}$ distilled water to preparation media of cultural. The 
assay were seeded with $5 \times 10^{5} \mathrm{cfu} / \mathrm{ml}$ of bacteria and $4 \times 10^{5} \mathrm{cfu} / \mathrm{ml}$ of fungi and filled with $(10 \mathrm{mg})$ powder samples for the tested bacteria and fungi, after solidification, they incubated at $37^{\circ} \mathrm{C}$ for 3 days, after which the diameter of the inhibition zones was measured.

\section{Results and Discussion}

\section{Tested microorganisms}

The antimicrobial activity of the prepared benzy- and/or styryl-1,2,4-triazine derivatives were determined against Escherichia coli (NCIM2065) as gram-negative bacteria, S. ureus as gram-positive bacteria and Candida albicans as fungi and the inhibition zones were measured in triplicates.

\section{Antimicrobial activity of some selected compounds:}

The antimicrobial activity of the prepared benzy- and/or styryl-1,2,4-triazine derivatives $(\mathbf{3 a}, \mathbf{5 b}, \mathbf{5 d}, \mathbf{8 b}, \mathbf{8 c}$ and 13) were determined against the tested organisms by standard methods using Cut plug method. 16,17 The inhibition zones were measured and tabulated in the following table.

Table (10): Antimicrobial activity of compounds (3a, 5b, 5d, 8b, 8c and 13).

\begin{tabular}{|l|l|l|l|}
\hline \multirow{2}{*}{ Comp } & \multicolumn{3}{|l|}{ Inhibition zone $(\mathrm{mm})$} \\
\cline { 2 - 4 } & $\begin{array}{l}\text { S.areous } \\
\text { MARSA }\end{array}$ & E. coli & C. albicans \\
\hline $\mathbf{3 a}$ & Negative & Negative & Negative \\
\hline $\mathbf{5 b}$ & 20 & Negative & 15 \\
\hline $\mathbf{5 d}$ & 25 & 14 & 10 \\
\hline $\mathbf{8 b}$ & 16 & Negative & Negative \\
\hline $\mathbf{8 c}$ & Negative & 7 & 20 \\
\hline $\mathbf{1 3}$ & Negative & 6 & Negative \\
\hline
\end{tabular}

In table 10: CompoundS 5b and 5d containing the amino phosphonates group. which might be the responsible group for the inhibitory effect on growth of the three tested microorganisms (S.areous MARSA, E. coli and $C$. albicans). compound. $\mathbf{8 b}$ showed antimicrobial ACTIVITY with S.areous MARSA, only. compounds 8C and 13 were tested against $E$. coli and $C$. albicans. compound . 8C showed antimicrobial activities against $E$. coli and $C$. albicans. Whiles Compound . 13 showed antimicrobial effect with E. coli only. Compound . 5d exhibited the highest inhibition zone.

\section{Determination of Minimal inhibitory concentrations (MICs)}

MICs of some synthetic compounds were determined for each antimicrobial agent by using agar dilution method ${ }^{\mathbf{1 8}}$. The inhibition zone was measured in triplicates in different concentrations $(0.5,1.0,2.0 \mathrm{ug} / \mathrm{ml})$ and the mean values of MICs are tabulated in the following table.
Table (11): (MICs $\boldsymbol{\mu g} / \boldsymbol{m l})$ of compounds (3a, $5 \mathrm{~b}, \mathbf{5 d}, \mathbf{8 b}$, 8c and 13).

\begin{tabular}{|l|l|l|l|}
\hline \multirow{2}{*}{ Comp } & \multicolumn{3}{l|}{ Inhibition zone $(\mathrm{mm})$} \\
\cline { 2 - 4 } & $\begin{array}{l}\text { S.areous } \\
\text { MARSA }\end{array}$ & E. coli & C. albicans \\
\hline $\mathbf{3 a}$ & - & - & - \\
\hline $\mathbf{5 b}$ & 2.0 & - & 1.0 \\
\hline $\mathbf{5 d}$ & 2.0 & 1.0 & 5.0 \\
\hline $\mathbf{8 b}$ & 0.5 & - & - \\
\hline $\mathbf{8 c}$ & - & 1.0 & 1.0 \\
\hline $\mathbf{1 3}$ & - & 1.0 & - \\
\hline
\end{tabular}

In table 11 the MICs of the above compounds were tested against S.areous MARSA, E. coli and C. albicans. Compound 5d yielded MICs $1 \mu \mathrm{g} / \mathrm{ml}$. It was found that the highest effective MICs was 5d. The above compounds exhibited MICs ranged between $0.5-5 \mu \mathrm{g} / \mathrm{ml}$.

\section{Conclusion}

In this paper. the authors reported the synthesis of some new 1,2,4-triazine derivatives to explore their biological activity. Preliminary results showed that some of them gave antibacterial activity.

\section{REFERENCES}

1- N. R. El-Brollosy, P. T. Jorgensen, B. Dahan, A-M. Boel, E. B. Pedersen and C. Nielsen, $J$. Med. Chem., 45, 5721 (2002).

2- C. O. Usifoh and G. K. E. Scriba, Arch. Pharm.(Weinheim.), 333, 261 (2000).

3- S. Plescia, G. Daiolone, L. Ceraulo, M. L. Bajardr and R. Reina, Farmaco ED. Sci., 39, 120 (1984).

4- N. B. EL-Dayaf, M.Sc. Thesis, Faculty of Science (Damietta) Mansoura Univ., Egypt (2004) under the supervision of A. A. ElBarbary, I. M. El-Sharkawy and A. ElSharabasy.

5- A. A. El-Barbary, M. A. Sakaran, A. M. ElMadani and C. Nielsen, J. Heterocycl. Chem., 42, 935 (2005).

6- E. D. Stecher, F. Dunn and E. Gelbutm., $J$. Am. Chem. Soc., 79, 4748 (1957).

7- J. S. Buck and W. S. Ide, Organic Synthesis, 11, John Wiley and Sons, Inc. New York, (1964).

8- N. A. Salahuddin, A. A. El-Barbary and N. I. Abdo, Polymer Composites, 1190 (2009). 
9- M. A. Badawy, S. A. Abdel. Hady, M. M. Eid and Y. A. Ibrahime, Chem. Ber., 117, 1083 (1984).

10-K. Tsuchiya, S.Kobayashi, T. Harada, T. Kurokawa, T. Nakagawa, N. Shimada and Kobayashi, J. Antibiot., 48, 626 (1995).

11 - E. S. H. El-Ashry and Y. El Kilany, Advances in Heterocyclic Chem.; A. R. Katritzky; Academic press; New York, 69, 129 (1998).

12-S. M. Lu and R. Y. Chen, Org. Prep. And Proc.Inter., 32(3), 302 (2000).

13- H. Bader and J. Downer, J. Chem. Soc., 1636 (1953).
14- A. A. El-Barbary, A. Z. Abou-El-Ezz, A. A. Abed-Kader,M.El-DalyandC. Nielsen,Phosphorus, Sulfur and Silicon, 179, 1497 (2004).

15- J.Okuda, K. Inagaki, I. Miwa and T. Yashiro, Chem. Pharm. Bull. ,Japan, 30 , 3244 (1982)

16- R. Mala and M. Sarojini, Cell and Tissue Res., 9, 1951 (2009).

17- J. Andrews, Antim. Chem., 48, 5-16 (2001).

18- P. Hsueh and J. Chang, Clin Micro., 35, 1021 (1997).

$$
\begin{aligned}
& \text { الملخص باللغة العربية } \\
& \text { أحمد أحمد البربري - أفراح محمد شرف بأه } \\
& \text { قسم الكيمياء - كلية العلوم - جامعة طنطا - ج .م.ع }
\end{aligned}
$$

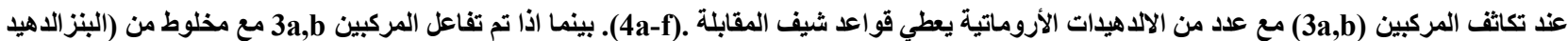

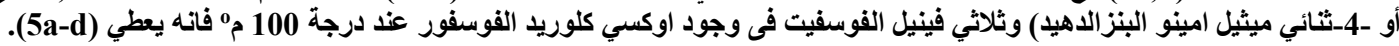

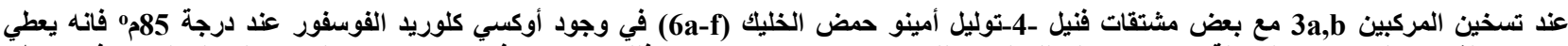

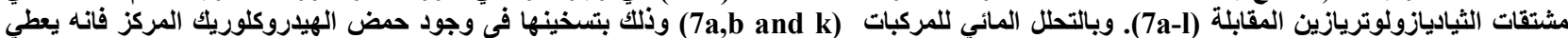
الأمينات الجديدة المقابلة (8a - ca عند غليان المركب 3a,b مع مشتقات البنزواوكزازون (9) فى حمض الخليك الثلجى فانه يعطي المركبيات (10a,b).

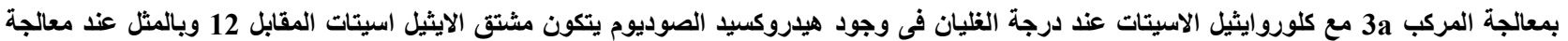

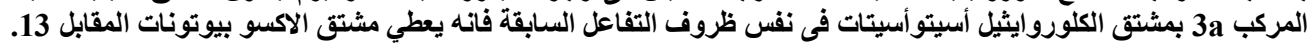

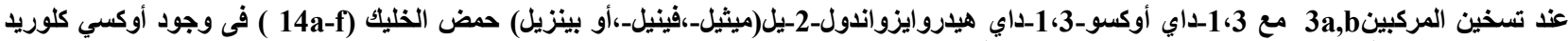

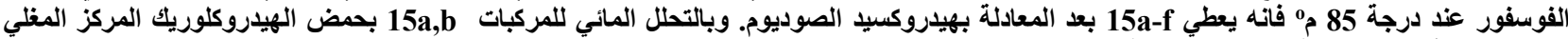

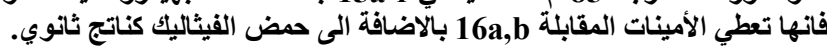

عند صهر المركبات 3a,b مع 1،-4- بيوتان سالتون عند درجة حرارة 180م0 فانه يعطي المركبات (18a,b) المقابلة. ولقد تم اختبار العديد من النواتج بيولوجيا كمضادات للبكتيريا بنوعيها الجرام (موجب) والجرام ( سالب) ووجد ان لبعضها نتائج ايجابية. 


\section{A. A. El-Barbary ${ }^{*}$ A. M. Sharaf,Bader E-Bader}

Delta J. Sci. 2016 ; Vol. 38 : (0-0) 


\section{A. A. El-Barbary ${ }^{*}$ A. M. Sharaf,Bader E-Bader}

Delta J. Sci. 2016 ; Vol. 38 : (0-0) 